\title{
Quiescent NIR and optical counterparts to candidate black hole $\mathrm{X}$-ray binaries
}

\author{
K. M. López, ${ }^{1,2 \star ~ P . ~ G . ~ J o n k e r, ~}{ }^{1,2 \star ~ M . ~ A . ~ P . ~ T o r r e s, ~}{ }^{1,3,4}$ M. Heida ${ }^{\odot}{ }^{5}$ A. Rau ${ }^{6}$ and \\ D. Steeghs ${ }^{7}$ \\ ${ }^{1}$ SRON Netherlands Institute for Space Research, Sorbonnelaan 2, NL-3584 CA Utrecht, the Netherlands \\ ${ }^{2}$ Department of Astrophysics/IMAPP, Radboud University, PO Box 9010, NL-6500 GL Nijmegen, the Netherlands \\ ${ }^{3}$ Instituto de Astrofísica de Canarias, E-38200 La Laguna, Tenerife, Spain \\ ${ }^{4}$ Departamento de Astrofísica, Universidad de La Laguna, Astrofísico Francisco Sánchez s/n, E-38206 La Laguna, Tenerife, Spain \\ ${ }^{5}$ Space Radiation Laboratory, California Institute of Technology, Pasadena, CA 91125, USA \\ ${ }^{6}$ Max-Planck-Institut für extraterrestrische Physik, Giessenbachstraße 1, D-85748 Garching, Germany \\ ${ }^{7}$ Department of Physics, University of Warwick, Coventry CV4 7AL, UK
}

Accepted 2018 October 12. Received 2018 October 12; in original form 2018 August 7

\begin{abstract}
We present near-infrared and optical imaging of 15 candidate black hole X-ray binaries. In addition to quiescent observations for all sources, we also observed two of these sources (IGR J17451-3022 and XTE J1818-245) in outburst. We detect the quiescent counterpart for 12 out of 15 sources, and for the remaining 3 , we report limiting magnitudes. The magnitudes of the detected counterparts range between $K_{\mathrm{s}}=17.59$ and $K_{\mathrm{s}}=22.29$ mag. We provide (limits on) the absolute magnitudes and finding charts of all sources. Of these 12 detections in quiescence, 7 detections represent the first quiescent reported values (for MAXI J1543-564, XTE J1726-476, IGR J17451-3022, XTE J1818-245, MAXI J1828-249, MAXI J1836-194, Swift J1910.2-0546), and 2 detections show fainter counterparts to XTE J1752-223 and XTE $\mathrm{J} 2012+381$ than previously reported. We used theoretical arguments and observed trends, for instance between the outburst and quiescent X-ray luminosity and orbital period $P_{\text {orb }}$ to derive an expected trend between $\Delta K_{\mathrm{S}}$ and $P_{\text {orb }}$ of $\Delta K_{\mathrm{S}} \propto \log P_{\mathrm{orb}}^{0.565}$. Comparing this to observations, we find a different behaviour. We discuss possible explanations for this result.

Key words: stars: black holes - infrared: stars - X-rays: individual: XTE J1818-245; IGR J17451-3022; XTE J1726-476; XTE J2012+381; XTE J1752-223; MAXI J1543-564.
\end{abstract}

\section{INTRODUCTION}

An X-ray binary (XRB) is defined as a system in which a neutron star (NS) or a black hole (BH) is accreting matter from its companion star. These systems can be classified according to the mass of the donor star, where we find high-mass X-ray binaries (HMXBs) and low-mass X-ray binaries (LMXBs). HMXBs are accreting mass through stellar winds or Roche lobe overflow from a massive $\operatorname{star}\left(M \geq 10 \mathrm{M}_{\odot}\right)$ with typical spectral types of $\mathrm{O}$ and $\mathrm{B}$ for main-sequence stars. The donor companion for LMXBs is typically of K-M spectral type low-mass star and has $M \leq 1 \mathrm{M}_{\odot}$, which transfers mass by Roche lobe overflow (Charles \& Coe 2003). XRBs with spectral type A-F donor stars have also been identified, commonly referred to as intermediate-mass XRBs (IMXBs; Pfahl, Rappaport \& Podsiadlowski 2003). These are thought to be the

^E-mail: k.m.lopez@sron.nl (KML); p.jonker@sron.nl (PGJ) progenitors of some LMXBs (Podsiadlowski, Rappaport \& Pfahl 2002).

Whether an XRB is a (semi) persistent source or shows outburst - quiescence cycles depends on the mass transfer rate and the orbital period $P_{\text {orb }}$ (King, Kolb \& Burderi 1996). Persistent sources typically have X-ray luminosities from 1 to 100 per cent of the Eddington limit, causing the accretion disc to dominate the optical spectrum, hiding the companion star in most cases, except a few giant star mass donors (e.g. Jonker et al. 2005; Steeghs et al. 2013). On the other hand, X-ray transients (XRTs) are characterized by episodic outbursts caused by mass transfer instabilities in the accretion disc (Dubus, Hameury \& Lasota 2001); between these outbursts, they decay back to the quiescent state $\left(L_{X}<10^{33} \mathrm{erg} \mathrm{s}^{-1}\right.$; e.g. Garcia et al. 1998; Lasota 2000; Jonker 2008; Guillot et al. 2009; Plotkin, Gallo \& Jonker 2013; Bernardini et al. 2016; Mata Sánchez et al. 2017), where the optical detection of the donor star is often, though not always (e.g. Torres et al. 2015), possible. This gives the opportunity to perform radial velocity measurements that allows us to study the orbital period evolution and to measure dynamically the 
mass of the compact accretor (Casares \& Jonker 2014). The latter is the best way to determine its nature since BH and NS systems display similar outburst properties such as hysteresis patterns in their hardness-intensity diagrams (Muñoz-Darias et al. 2014), though the $\mathrm{X}$-ray timing properties do show differences between NS and $\mathrm{BH}$ accretors (see for instance the overview of Lewin \& van der Klis 2010). It is also possible to use the radio properties as BHs are more radio-loud than NSs (Fender \& Kuulkers 2001; Fender, Gallo \& Jonker 2003).

XRTs are often first detected at X-ray wavelengths in outburst thanks to all-sky X-ray monitoring programmes, for instance through the RXTE satellite before 2012 (Bradt, Rothschild \& Swank 1993), INTEGRAL (Kuulkers et al. 2007), Neil Gehrels Swift Observatory (Krimm et al. 2013), and MAXI (Matsuoka et al. 2009) missions. Until today, we have 19 dynamically confirmed Galactic stellar-mass BHs (Casares \& Jonker 2014 and references therein, Mata Sánchez et al. 2015, Tetarenko et al. 2016b), where 18 of these are in L/IMXBs and one in an HMXB (Cyg X-1, Orosz et al. 2011b). There is a concentration towards the Galactic bulge and plane in the spatial distribution of these sources (i.e. $340^{\circ}<l<20^{\circ}$ and $\|b\|<$ $10^{\circ}$, e.g. Jonker \& Nelemans 2004). Around 50 per cent of these, 19 sources are located within about $4.5 \mathrm{kpc}$ from the Sun, which indicates that the dynamical mass measurements are challenging observationally due to the faintness of the mass donor stars, in part due to the sometimes high interstellar extinction towards sources located in the plane/bulge regions. Additionally, about 40 black hole $\mathrm{X}$-ray binary $(\mathrm{BHXB})$ candidates are known, which even though they do not have an estimate for their mass, present similar outburst properties to already classified BHXBs (see Belloni, Motta \& Muñoz-Darias 2011 for a review). The number of BHXB candidates grows at an approximate rate of 2 objects per year (Corral-Santana et al. 2016).

As mentioned before, the quiescent state of XRTs is the ideal starting point to perform dynamical studies of these binary systems. The more dynamically confirmed black holes are known, the more information we have about the mass distribution of BHs in our Galaxy. This is important for understanding the physics of supernova explosions, the equation of state of nuclear matter (Casares, Jonker \& Israelian 2017) and the survival of interacting binaries, including those that might eventually merge and produce bursts of gravitational wave radiation (e.g. Abbott et al. 2016a,b). The mass distribution of BHs is expected to be smooth (Fryer \& Kalogera 2001); however, observations have shown a gap between NSs and BHs in the range of $2-5 \mathrm{M}_{\odot}$ (Özel et al. 2010; Farr et al. 2011). The existence of the gap is still under debate. Some argue that the observed distribution may be biased by selection effects (i.e. Özel et al. 2010) and biases in the mass measurement procedure. For instance, Kreidberg et al. (2012) claim that there might be a systematic trend in the inclination angle determinations that lead to an underestimate of the inclination, and thus, an overestimate of the BH mass (see also van Grunsven et al. 2017). Others state that the gap is real and could shed light on supernovae explosion models (i.e. Belczynski et al. 2012; Fryer et al. 2012). With Gaia (Gaia Collaboration et al. 2016), we will be able to obtain accurate distances and proper motions for several XRBs, necessary to determine $\mathrm{BH}$ natal kicks (e.g. van Paradijs \& White 1995; Jonker \& Nelemans 2004; Miller-Jones et al. 2009; Reid et al. 2011, 2014; Repetto \& Nelemans 2015; Mandel 2016) and hence, constrain the formation and evolution of BHXBs.

In this paper, we report the quiescent magnitudes, or upper limits, of the counterparts to 15 BHXB candidates in the near-infrared (NIR) and optical bands. For seven of them our measurements are the first reported to date. We provide also outburst magnitudes for two of these 15 sources (XTE J1818-245 and IGR J17451-3022); moreover, we detected for the first time the counterpart for IGR J17451-3022. We describe our sample in Section 2, the observations in Section 3 and the data reduction and analysis in Section 4. Our results are presented in Section 5 and discussed in Section 6, and we end with the conclusions of our work in Section 7. We report values taken from literature as found in the original work, i.e. if uncertainties were originally not given, we report the value without uncertainties (this includes points without error bars in our plots).

\section{SAMPLE}

Our sample consists of 15 BHXB candidates (see Table 1 for discovery dates, coordinates, and distances). With the exception of MAXI J1957+032 and MAXI J1807+132, the sources can be found in either the BlackCAT (Corral-Santana et al. 2016) or WATCHDOG (Tetarenko et al. 2016a) catalogues for BHXB candidates. Both MAXI J1957+032 and MAXI J1807+132 are NS candidates (Ravi 2017; Shidatsu et al. 2017a) reported after we had observed them; however, their spectral features are also consistent with them being BH XRTs as no type I X-ray bursts or pulsation have been found (Mata Sánchez et al. 2017; Munoz-Darias et al. 2017; Shidatsu et al. 2017b). Thirteen sources are located at latitudes $\|b\|<7^{\circ}$, whereas MAXI J1957+032 is at $b \sim 13^{\circ}$ and MAXI $\mathrm{J} 1807+132$ is at $b \sim 15^{\circ}$. All 15 sources are observed during quiescence: 7 sources in the $K_{\mathrm{s}}$ band (XTE J2012+381, XTE J1752-223, Swift J174510.8-262411, MAXI J1828-249, MAXI J1836-194, MAXI J1957+032, and XTE J1856+053), XTE J1726-476 in the $J$ and $K_{\mathrm{s}}$ bands, MAXI J1543-564 in the $H$ and $K_{\mathrm{s}}$ bands, MAXI $\mathrm{J} 1807+132$ in the $i^{\prime}$ band, Swift $1910.2-0546$ in the $K_{\mathrm{s}}, r^{\prime}, i^{\prime}$ bands and two sources (XTE J1650-500 and Swift J1539.2-6227) in the $g^{\prime}, r^{\prime}, i^{\prime}, z^{\prime}, J, H, K_{\mathrm{s}}$ bands. XTE J1818-245 and IGR J17451-3022 are observed both in quiescence and in outburst, both in the $K_{\mathrm{s}}$ band (see Table 2).

\section{OBSERVATIONS}

We obtained NIR and optical observations for this project using five different telescopes. To acquire $J H K_{\mathrm{s}}$-band images, we used the William Herschel Telescope with the Long-slit Intermediate Resolution Infrared Spectrograph (WHT/LIRIS), the Keck I Telescope on Mauna Kea with the Multi-Object Spectrometer for Infra-Red Exploration (Keck/MOSFIRE, McLean et al. 2010, 2012), the Walter Baade Magellan Telescope with the Persson's Auxiliary Nasmyth Infrared Camera (Magellan/PANIC, Martini et al. 2004), and the Very Large Telescope Unit 4 with the High Acuity Wide field $K$-band Imager (VLT/HAWK-I, Pirard et al. 2004). To obtain the optical ( $r^{\prime} i$ band) data, we used the William Herschel Telescope with the auxiliary-port camera (WHT/ACAM). Finally, we got NIR and optical observations in seven filters $\left(g^{\prime}, r^{\prime}, i^{\prime}, z^{\prime}, J, H, K_{\mathrm{s}}\right)$ using the Gamma-Ray Burst Optical/Near-Infrared Detector (MPI/GROND, Greiner et al. 2008) at the MPI/ESO $2.2 \mathrm{~m}$ telescope at the ESO La Silla Observatory.

LIRIS has a pixel scale of 0.25 arcsec pixel ${ }^{-1}$ and a field of view of 4.27 arcsec $\times 4.27$ arcsec; MOSFIRE provides a pixel scale of 0.18 arcsec pixel ${ }^{-1}$ and a field of view of $6.1 \mathrm{arcmin} \times 6.1 \mathrm{arcmin}$; and PANIC has a pixel scale of $0.125 \operatorname{arcsec}_{\text {pixel }}^{-1}$ and a field of view of 2.0 arcmin $\times 2.0$ arcmin. HAWK-I provides a pixel scale of 0.106 arcsec pixel ${ }^{-1}$ and a field of view of $7.5 \operatorname{arcmin} \times$ 
Table 1. Discovery date, position and, when constrained, distance to the BHXBs which are the subject of this paper.

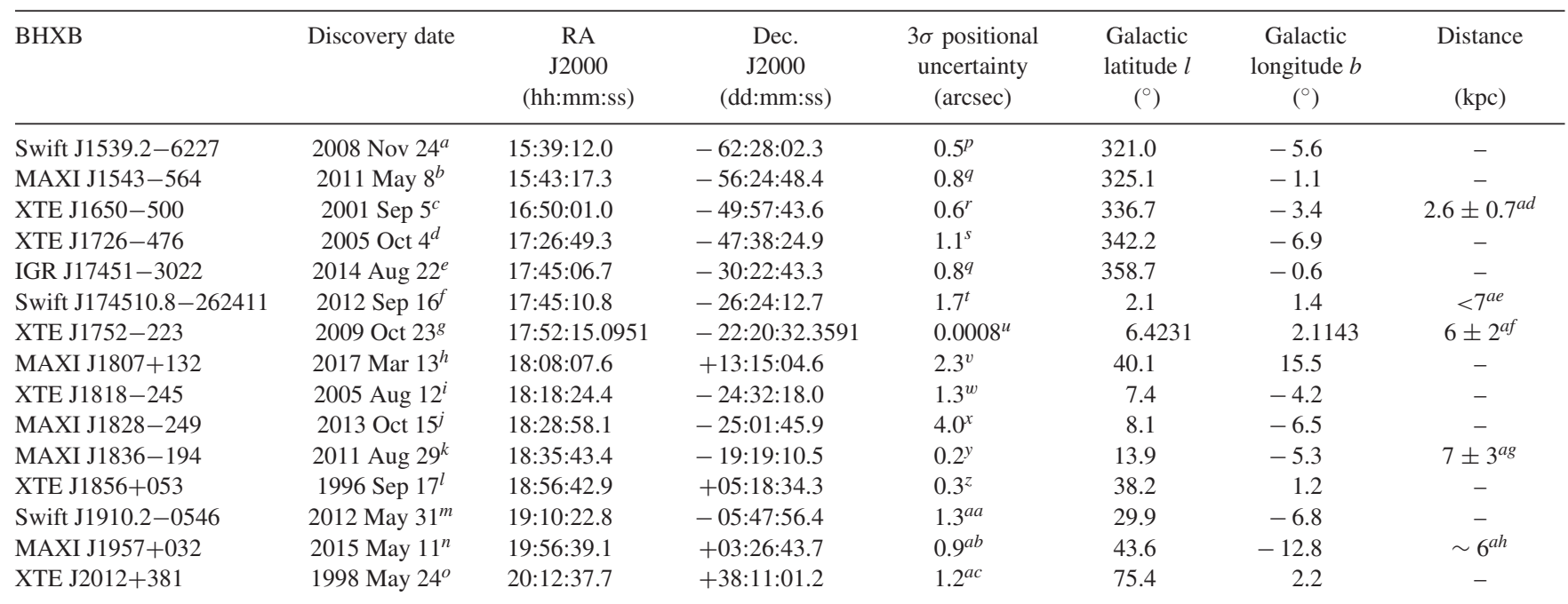

References: ${ }^{a}$ Krimm et al. (2008), ${ }^{b}$ Negoro et al. (2011a), ${ }^{c}$ Miller (2001), ${ }^{d}$ Turler et al. (2005), ${ }^{e}$ Chenevez et al. (2014), ${ }^{f}$ Cummings et al. (2012), ${ }^{g}$ Markwardt et al. (2009), ${ }^{h}$ Negoro et al. (2017), ${ }^{i}$ Levine et al. (2005a), ${ }^{j}$ Nakahira et al. (2013), ${ }^{k}$ Negoro et al. (2011b), ${ }^{l}$ Marshall et al. (1996), ${ }^{m}$ Usui et al. (2012), ${ }^{n}$ Negoro et al. (2015a); Cherepashchuk et al. (2015), ${ }^{o}$ Remillard et al. (1998), ${ }^{p}$ Krimm et al. (2011), ${ }^{q}$ Chakrabarty, Jonker \& Markwardt (2014), ${ }^{r}$ Tomsick, Kalemci \& Kaaret (2004), ${ }^{s}$ Maitra et al. (2005), ${ }^{t}$ Rau et al. (2012b), ${ }^{u}$ Miller-Jones et al. (2011a), ${ }^{v}$ Kennea et al. (2017a), ${ }^{w}$ Rupen, Dhawan \& Mioduszewski (2005), ${ }^{x}$ Kennea et al. (2013), ${ }^{y}$ Russell et al. (2015), ${ }^{z}$ Torres et al. (2007), ${ }^{a}$ Rau, Greiner \& Schady (2012a), ${ }^{a b}$ Rau, Yates \& Greiner (2015), ${ }^{a c}$ Hynes et al. (1998), ${ }^{a d}$ Homan et al. (2006). ${ }^{a e}$ Muñoz-Darias et al. (2013), ${ }^{a f}$ Ratti et al. (2012), ${ }^{a g}$ Russell et al. (2014), and ${ }^{a h}$ Mata Sánchez et al. (2017).

$*$ The coordinates for the counterpart that we identified are RA $=17: 45: 06.654$, Dec. $=-30: 22: 43.67$.

7.7 arcmin ACAM has a pixel scale of 0.25 arcsec pixel $^{-1}$ and a circular field of view with a diameter of 8.0 arcmin and GROND provides a pixel scale in the optical(NIR) channels of 0.158 arcsec (0.60 arcsec) pixel $^{-1}$, and a field of view in the optical(NIR) channel of $5.4 \operatorname{arcmin} \times 5.4 \operatorname{arcmin}(10 \operatorname{arcmin} \times 10 \operatorname{arcmin})$. The NIR observations with LIRIS, PANIC, and MOSFIRE were performed using multiple repetitions of a 5-position-dither pattern where several images were taken at each dither position, while the HAWK-I observations $^{1}$ were done with 8 pointings of $60 \mathrm{~s}$ each for the $H$ band and 7 pointings of $60 \mathrm{~s}$ each for the $K_{\mathrm{s}}$ band. The optical observation with WHT/ACAM was done with 6 different pointings of $300 \mathrm{~s}$ each; additionally, the GROND observations ${ }^{2}$ in the $g^{\prime}$, $r^{\prime}, i^{\prime}, z^{\prime}$ filters were done with 8 pointings of $62 \mathrm{~s}$ each at different offset positions, while the observations in the $J, H, K_{\mathrm{s}}$ filters were done with 48 pointings of $10 \mathrm{~s}$, dithered only for the $K_{\mathrm{s}}$ band and at different positions for the $J, H$ bands.

We targeted two sources in the $K_{\mathrm{s}}$ band with PANIC: XTE J1818-245 was observed in outburst in 2005 September and then in quiescence in 2006 May, and XTE J1726-476 was observed in quiescence in 2006 August in both $J$ and $K_{\mathrm{S}}$ bands. Five sources were observed in the $K_{\mathrm{s}}$ band with LIRIS: IGR J17451-3022 was observed in outburst in 2015 April and then in quiescence in 2016 March, and four other sources (XTE J2012+381, XTE J1752-223, Swift J174510.8-262411, and Swift J1910.2-0546) were observed in quiescence in 2017 April. The last source, Swift J1910.2-0545, was also observed in quiescence with ACAM in both $r^{\prime}$ and $i^{\prime}$ bands, in 2015 July. We took $K_{\mathrm{s}}$-band images with Keck/MOSFIRE for four sources (MAXI J1828-249, MAXI J1836194, MAXI J1957+032, and XTE J1856+053) in quiescence in June 2017, MAXI J1807+132 was observed in quiescence with ACAM in the $i^{\prime}$ band in July 2017, two sources in quiescence

${ }^{1}$ ESO programme 1101.D-0105(A), PI: P.G. Jonker.

${ }^{2}$ ESO programme 099.A-9025(A), PI: A. Rau.
(XTE J1650-500 and Swift J1539.2-6227) were observed with GROND in September 2017, and finally, MAXI J1453-564 was observed in quiescence in both $H$ and $K_{\mathrm{s}}$ bands with HAWK-I in 2018 April. For a full observing log see Table 2, where also the average seeing is provided, as a measure of the image quality during the observations.

\section{DATA REDUCTION AND ANALYSIS}

\subsection{Data reduction}

For the images taken with GROND, the data reduction was done with the standard tools and methods described in Krühler et al. (2008). The PANIC data were processed with IRAF scripts implemented by Martini et al. (2004) for the reduction of data from that instrument. These procedures included dark and flat-field correction as well as sky subtraction. In the data reduction process, the raw frames were first dark subtracted. Master flat-fields were built by combining twilight flat-field frames scaled by the mode and these were applied to the target images. A sky image was made by masking out stars from each set of dithered target frames and was subtracted from the associated set of frames. Finally, a single target image was obtained by average combining the sky-subtracted images.

The LIRIS, ACAM, MOSFIRE, and HAWK-I data were reduced using the data reduction software THELI (Schirmer 2013). In order to flat-field correct the NIR data, we produced a master flat with THELI by median combining the sky flat images taken during twilight. Additionally, a sky background model is generated by median combining the observations without correcting for the offsets introduced by the dithering, which then is subtracted from the individual data frames. After this, THELI detects sources in the images using SEXTRACTOR (Bertin \& Arnouts 1996) and obtains astrometric solutions with SCAMP (Bertin 2006). The astrometric 
Table 2. Observing log of the BHXB candidates discussed in this paper. All of the sources are observed in quiescence. An (*) marks sources also observed in outburst.

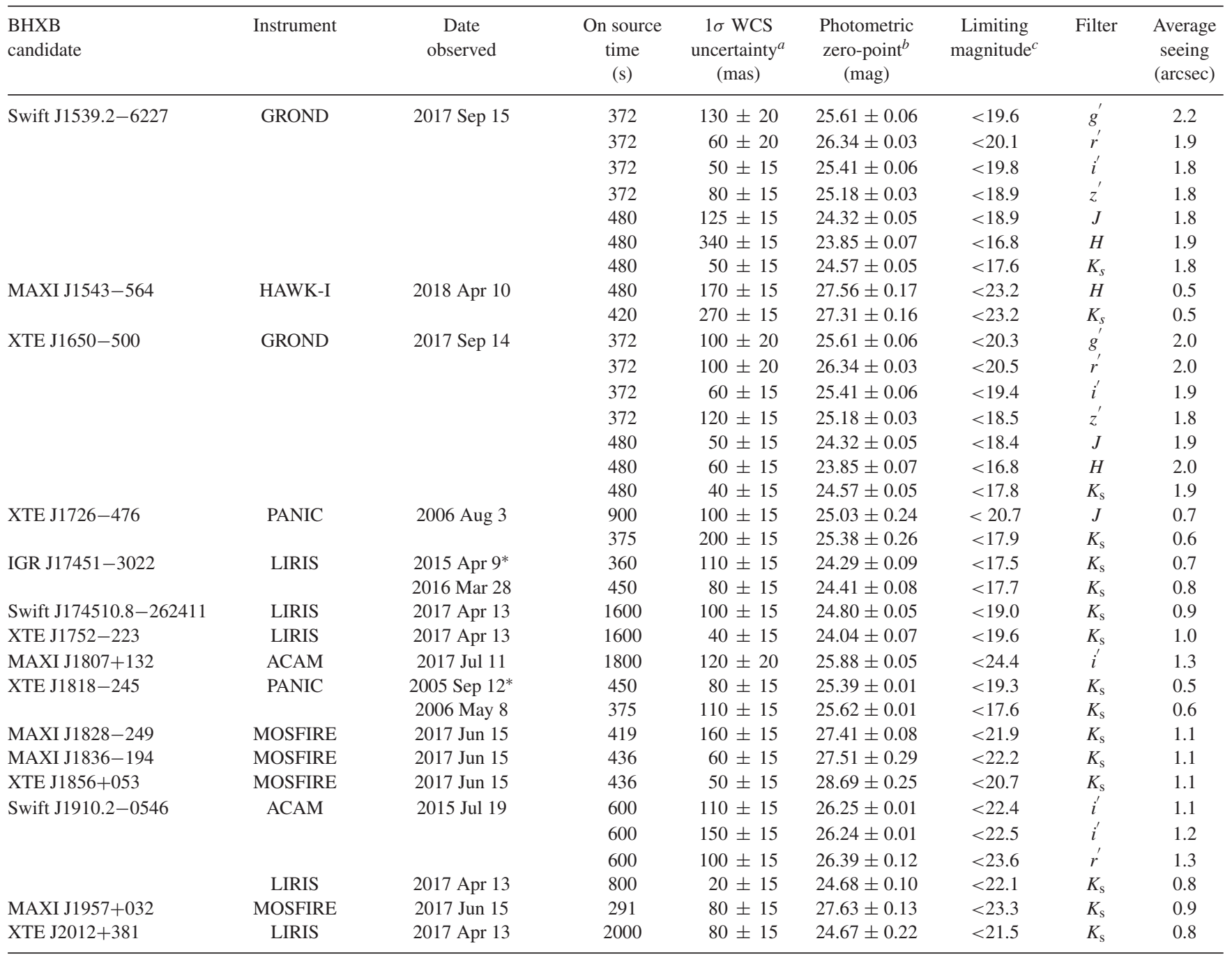

Note. *Observation in outburst. ${ }^{a}$ Uncertainty with respect to the reference catalog. The first value is a statistical uncertainty, given by the STARLINK GAIA tool, while the second value is a systematic uncertainty, and corresponds to the astrometric accuracy of the reference catalog (20 mas for UCAC4 and 15 mas for 2MASS). ${ }^{b}$ Photometric zero-point derived from the data with respect to the 2MASS catalogue, or VVV when possible, (for all the NIR sources), and the Pan-STARRS 1 catalogue (for the optical sources). ${ }^{c}$ Limiting magnitude close to the position of the BHXB.

solution is obtained by matching the detected positions to sources from different catalogues. The global astrometric solution is then used for the coaddition of all the frames using SWARP (Bertin et al. 2002).

\subsection{Astrometry}

To improve the accuracy of the global astrometric solution of the coadded images, we used the STARLINK tool GAIA, fitting at least five star positions from the 2 Micron All Sky Survey (2MASS; Skrutskie et al. 2006) or from the fourth US Naval Observatory CCD Astrograph Catalog (UCAC4; Zacharias et al. 2013) in order to build a local astrometric solution around the position of the sources. The rms errors of these fits are indicated in Table 2 as WCS (World Coordinate System) uncertainties, where the intrinsic systematic error of the catalogue with respect to the International Celestial Reference System (ICRS) is also listed (15 mas for 2MASS and 20 mas for UCAC4).

\subsection{Photometry}

For seven sources (Swift J1910.2-0546, XTE J1726-476, MAXI J1807+132, XTE J1752-223, MAXI J1543-564, Swift J1539.2-6227, and XTE J1650-500), we used SEXTRACTOR for the source detection and photometry, making sure that each detection was more than $3 \sigma$ above the local background. We performed aperture photometry to determine instrumental magnitudes. We determined the full width at half-maximum of point-like objects in each image with the STARLINK tool GAIA and we used it as the aperture size. We performed point spread function (PSF) photometry for the eight remaining images using the DAOPHOT package in IRAF $^{3}$ because the source density warrants this. The photometric zero-

\footnotetext{
${ }^{3}$ IRAF is distributed by the National Optical Astronomy Observatories, which are operated by the Association of Universities for Research in Astronomy, Inc., under cooperative agreement with the National Science Foundation.
} 
points of our NIR images were measured by using isolated 2MASS objects (or $\mathrm{VVV}^{4}$ objects, when possible) in the field of view, and for our optical images, we used isolated Panoramic Survey Telescope and Rapid Response System 1 (Pan-STARRS 1, Chambers et al. 2016; Flewelling et al. 2016) objects. We calibrated the instrumental magnitudes of those isolated sources with the magnitudes reported by their respective surveys, thus calculating the photometric zero-points (see Table 2). We ignore the colour term in our calibrations, as this term is small compared to other uncertainties in the photometry (Augusteijn 1992). Instrumental magnitudes for all detected sources were converted to apparent magnitudes using these zero-points. It should be noted that the formal uncertainty in the 2MASS/Pan-STARRS 1 photometric calibration that we employ is the largest contributor (90-95 percent) to the uncertainty in the apparent magnitudes. For the images in which we did not detect a counterpart, we estimated the limiting magnitude by simulating 10000 stars at the position of the XRB using the IRAF task MKOBJECTS and then detecting them with the task DAOFIND. We use the faintest magnitude at which the objects were detected at the $3 \sigma$ level as our limiting magnitude. For the five sources (XTE J1752-223, Swift J174510.8-262411, MAXI J1836-194, MAXI J1957+032, and XTE J1650-500) for which we determine a limiting absolute magnitude, we assumed $R_{V}=3.1$ (Fitzpatrick 1999), $R_{K_{\mathrm{s}}}=0.306$ (Yuan, Liu \& Xiang 2013), and $N_{\mathrm{H}}=1.87 \times 10^{21}$ atoms $\mathrm{cm}^{-2}$ $\mathrm{mag}^{-1} A_{V}$ (Bohlin, Savage \& Drake 1978) to correct for extinction. We use the distances indicated in Table 1, and although these estimates have large uncertainties (one value is a lower limit), even an underestimate of the distance by a factor of 2 would only result in a difference of $1.5 \mathrm{mag}$ in absolute magnitude. This is the difference between spectral types $\mathrm{K} 0 \mathrm{~V}$ and $\mathrm{K} 7 \mathrm{~V}$, or between M0V and M2V. All the magnitudes are given in the $\mathrm{AB}$ system, unless indicated otherwise; the conversion between the Vega and $\mathrm{AB}$ systems was done following Blanton $\&$ Roweis (2007), i.e. $J_{\mathrm{AB}}=J_{\mathrm{VEGA}}+0.91$, $H_{\mathrm{AB}}=H_{\mathrm{VEGA}}+1.39, K_{\mathrm{sAB}}=K_{\mathrm{sVEGA}}+1.85$.

\section{RESULTS}

\subsection{Sources observed in outburst}

\subsubsection{IGR J17451-3022}

This source was discovered by INTEGRAL/JEM-X on $2014 \mathrm{Au}-$ gust 22 (Chenevez et al. 2014). Suzaku observations done in 2014 September revealed eclipses in the light curve of IGR J17451-3022 (Jaisawal et al. 2015), from which they estimated a $P_{\text {orb }}$ of $\sim 6.3 \mathrm{~h}$. We performed an NIR observation of IGR J17451-3022 during its outburst and detected two sources (labelled A and B in Fig. 1a) in the 99.7 per cent confidence radius around the X-ray position. The source returned to quiescence in 2015 May (Bahramian et al. 2015). We observed it for a second time using the same telescope and instrument and filter on 2016 March 28 and detected again the same two sources (see Fig. 1b) in the 99.7 per cent confidence radius around the X-ray position (Chakrabarty et al. 2014). As is visible in Figs 1(a) and (b), these two sources are close to a bright star (2MASS J17450681-3022456, $K_{\mathrm{s}}=13.16$ ). To estimate the apparent magnitudes of the fainter sources minimizing contamination of this bright star, we subtracted it and then performed PSF photometry on the image. Then, using differential photometry we found that the apparent magnitude of the source labelled

${ }^{4}$ VISTA Variables in the Via Lactea survey (Minniti et al. 2010).
$\mathrm{B}$ varied between outburst and quiescence at the level of $7.8 \sigma$ (see Fig. 2).

The outburst and quiescent magnitudes are $K_{\mathrm{s}}=17.39 \pm 0.16$ and $K_{\mathrm{s}}=17.59 \pm 0.14$, respectively (see Table 3 ). Whereas these values are the same within the errors, the formal uncertainty in the 2MASS photometric calibration that we employ is the largest contributor to the uncertainty ( $0.13 \mathrm{mag}$ in both cases). Our differential photometry where we calibrate one frame against the magnitudes of the second shows that the source in quiescence is significantly fainter by $7.8 \sigma$. Given that the source is eclipsing (Jaisawal et al. 2015), the projected surface area of the accretion disc is small, possibly resulting in a similar magnitude for any combination of disc plus star during outburst and quiescence.

\subsubsection{XTE J1818-245}

The X-ray transient XTE J1818-245 was discovered on $2005 \mathrm{Au}$ gust 12 (Levine et al. 2005a) with the RXTE all-sky monitor. Steeghs et al. (2005a) observed it a few days after the outburst and detected an optical candidate counterpart with $R \sim 17.71$ mag that was later confirmed by Zurita Heras et al. (2011). We observed this source a month after the outburst discovery and detected a NIR counterpart with an apparent magnitude of $K_{\mathrm{s}}=16.18 \pm 0.02$; this is consistent with the result from Cadolle Bel et al. (2009). They observed XTE J1818-245 in the NIR 2 months after the outburst start and detected a counterpart with apparent magnitude $K=16.9 \pm 0.2$. We observed this source also during quiescence, approximately $10 \mathrm{yr}$ after the outburst ended, and we detected the NIR counterpart as well, with $K_{\mathrm{s}}=19.99 \pm 0.22$ (see Table 3 ). As can be seen from Figs 3(b) and (a), it is evident that the NIR source is bright during outburst and fades during quiescence. Hence, this is the quiescent NIR ( $K_{\mathrm{s}}$ band) counterpart to XTE J1818-245.

\subsection{Sources observed in quiescence}

\subsubsection{Swift J1539.2-6227}

Swift J1539.2-6227 was discovered on 2008 November 24 by Swift/BAT (Krimm et al. 2008). UV/optical observations made a month later revealed a counterpart with magnitudes of $u v w 2=18.07 \pm 0.03$ and $u v m 2=17.96 \pm 0.04$ (Krimm et al. 2009), which continued until at least until 2009 March (Torres et al. 2009a). The optical spectrum of the counterpart revealed no Balmer lines, nor evidence for HeII $4686 \AA$ or Bowen blend emission (Torres et al. 2009a). We observed this source with GROND and did not detect the counterpart down to limiting magnitudes of $g^{\prime}>19.6, r^{\prime}$ $>20.1, i^{\prime}>19.8, z^{\prime}>18.9, J>18.9, H>16.8$, and $K_{\mathrm{s}}>17.6$ (see Table 3).

\subsubsection{MAXI J1543-564}

Negoro et al. (2011a) discovered this source on 2011 May 8. UV/optical observations with Swift revealed no counterpart to this source down to limiting magnitudes of $V>19.47$ and $U>20.85$ (Kennea et al. 2011a), which is consistent with the large absorption column towards this source. Russell et al. (2011) detected a possible counterpart with a magnitude of $i=19.5$. Two days after the outburst was reported, Rau et al. (2011b) detected the same optical counterpart (called source A) with GROND, and reported outburst magnitudes of $g^{\prime}>22.8, r^{\prime}=20.7 \pm 0.1, i^{\prime}=19.4 \pm 0.1, z^{\prime}=$ $18.7 \pm 0.1, J=17.1 \pm 0.2, H=16.8 \pm 0.2$, and $K_{\mathrm{s}}=17.0 \pm 0.2$. Additionally, Rau et al. (2011b) reported the detection of two other 


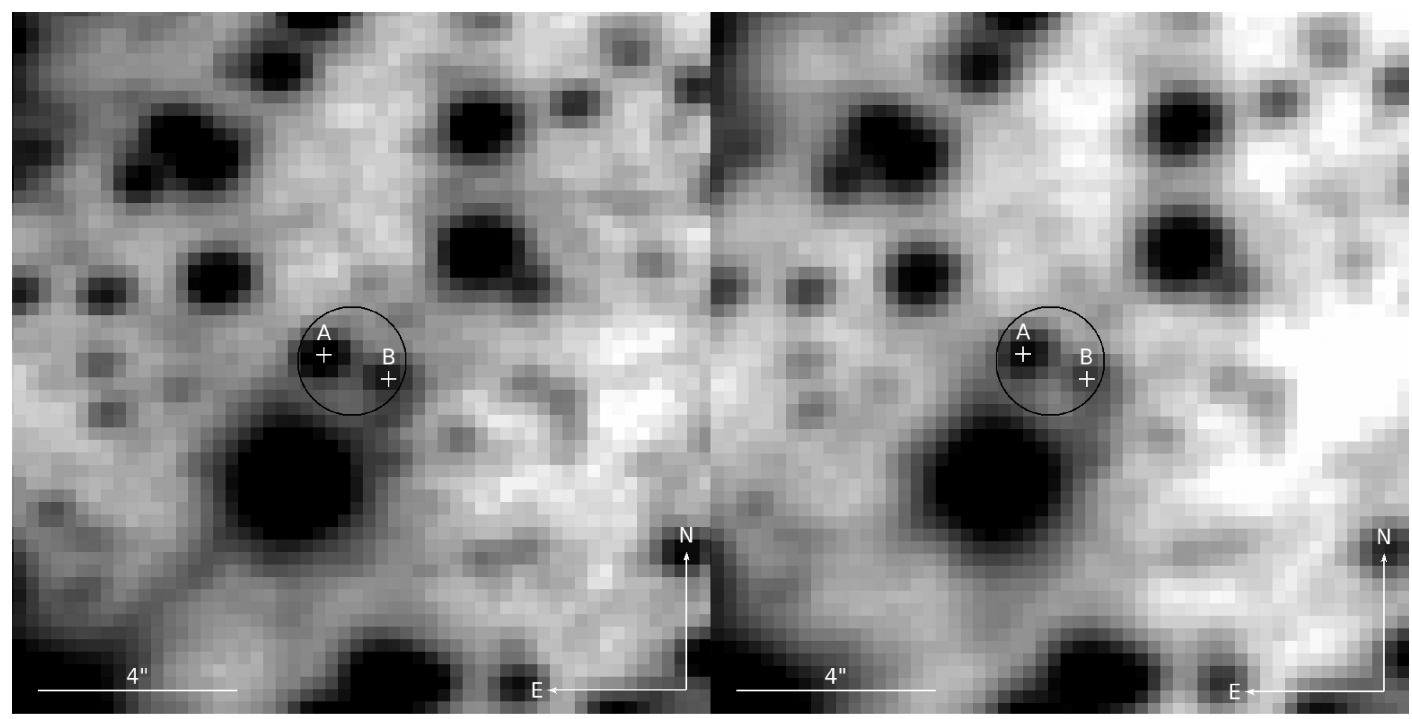

(a) IGR J17451 in outburst

(b) IGR J17451 in quiescence

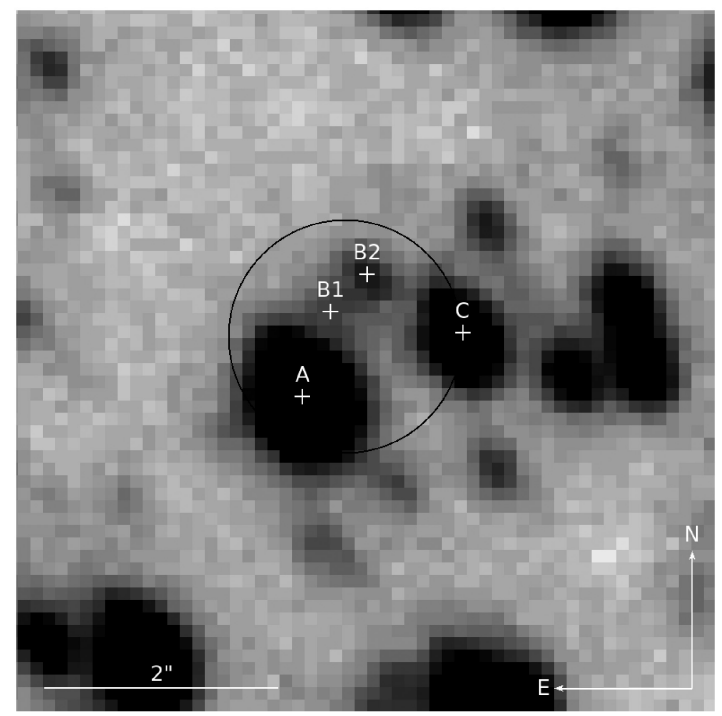

(c) MAXI J1543-564

Figure 1. Finder charts of the two XRBs for which the outburst counterpart has not been identified until now, so we have to rely on their Chandra X-ray localisation for the identification of the counterpart in quiescence. Upper panel: LIRIS $K_{\mathrm{S}}$-band image of IGR J17451-3022 in (a) outburst and (b) quiescence. As no counterparts were reported previously for IGR J17451-3022, we analysed the two sources (labelled A and B) inside the 99.7 per cent confidence region around the Chandra X-ray position (Chakrabarty et al. 2014), and using differential photometry, determined that the likely counterpart is source B as that source is fainter in quiescence than in outburst. Bottom panel: HAWK-I $K_{\mathrm{s}}$-band image of MAXI J1543-564, where we indicate the sources identified by Rau et al. (2011b) and label the in the same way as they did, with the difference that they identified one source B, whereas we detect two sources in this position (B1 and B2). These two sources are consistent with the position of the likely counterpart, according to a Chandra localization of the X-ray source (Chakrabarty, Jonker \& Markwardt 2011).

sources, called sources B and C, with magnitudes $z^{\prime} \sim 20.7$ and $z^{\prime}$ $\sim 20.5$, respectively. No measurements in the $J H K_{s}$ bands are reported, as they are strongly affected by the blending of sources A, B, and C. A Chandra localization of MAXI J1543-564 (Chakrabarty et al. 2011) showed that the optical source detected by Russell et al. (2011) and Rau et al. (2011b) was not likely the counterpart but instead, the source identified as source B by Rau et al. (2011b) is a better candidate counterpart. We observe this source with HAWK-I and detect two sources inside the $3 \sigma$ confidence radius around the position of source B from Rau et al. (2011b) in both $H$ and $K_{\mathrm{s}}$ bands (see Fig. 1c). We label the sources B1 and B2 and estimate magnitudes of $H=20.5 \pm 0.2$ and $K_{\mathrm{s}}=20.68 \pm 0.16$ for B1 and $H=21.7 \pm 0.2$ and $K_{\mathrm{s}}=21.8 \pm 0.2$ for B2. Further observations are required in order to determine which one is the true counterpart (if any).

\subsubsection{XTE J1650-500}

This XRT was discovered on 2001 September 5 by RXTE and optical observations revealed a counterpart with $V=17$ (CastroTirado et al. 2001). A month later, with observations from ESO NTT, NIR/optical magnitudes were derived (Curran, Chaty \& Zurita 


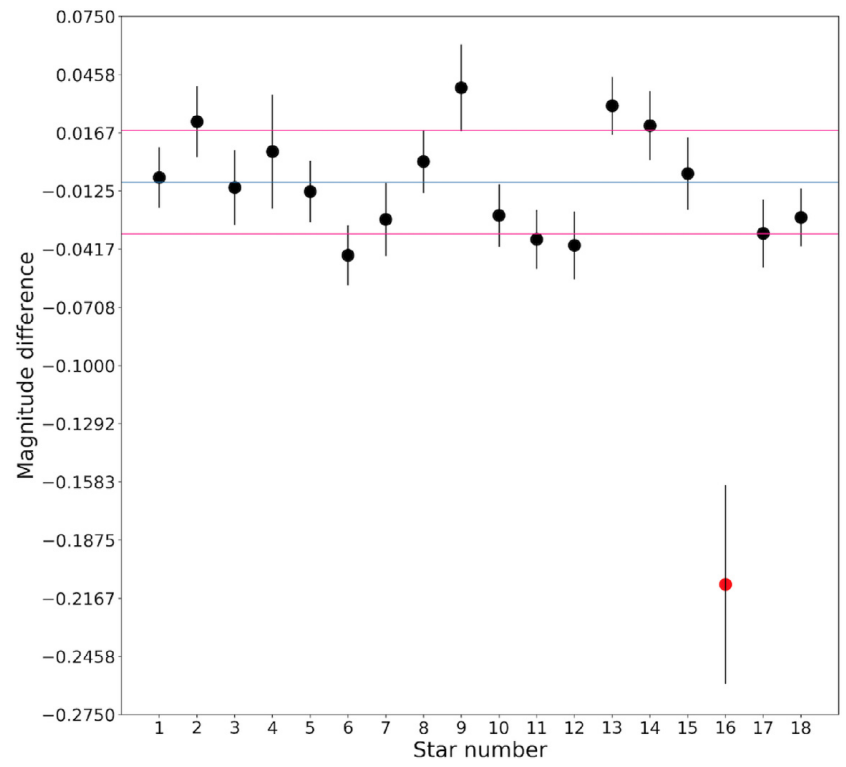

Figure 2. Magnitude difference of 18 stars inside a radius of $\sim 40$ arcsec around the Chandra X-ray position of IGR J17451-3022, between its outburst and quiescence images. The blue line indicates the average of all the differences and the pink line indicates the standard deviation of the distribution. Note how source B (red symbol) is clearly an outlier.

Heras 2012): $V=17.1 \pm 0.2, R=16.9 \pm 0.4, I=16.14 \pm 0.13$, $J=15.33 \pm 0.11, H=15.18 \pm 0.11$, and $K_{\mathrm{s}}=15.14 \pm 0.13 \mathrm{mag}$. It was observed $\sim 10$ months later (2002 August 10) in the optical with the Magellan telescope, and the counterpart was detected in quiescence, with magnitudes of $R \sim 22$ and $V \sim 24$ (Garcia \& Wilkes 2002). We observed this source with GROND and did not detect the counterpart down to limiting magnitudes of $g^{\prime}>20.3, r^{\prime}$ $>20.5, i^{\prime}>19.4, z^{\prime}>18.5, J>18.4, H>16.8$, and $K_{s}>17.8$ (see Table 3), resulting in an outburst amplitude of $>2$ mag in the $J H K_{s}$ bands. With a distance of $d=2.6 \pm 0.7 \mathrm{kpc}$ given by Homan et al. (2006) and $N_{\mathrm{H}}=0.5 \pm 0.1 \times 10^{22} \mathrm{~cm}^{-2}$ (Miniutti, Fabian \& Miller 2004), we calculate an extinction of $A_{K_{s}}=0.270$, which gives a limiting absolute magnitude of $M_{K}>5.4$, making the donor star an MOV spectral type or later.

\subsubsection{XTE J1726-476}

XTE J1726-476 was discovered with RXTE by Levine, Lin \& Remillard (2005b) on 2005 October 4. Optical I-band observations revealed a counterpart with $I=17.42 \pm 0.11$ mag days after the outburst start (Maitra et al. 2005). Additionally, Steeghs et al. (2005b) observed XTE J1726-476 in the NIR and detected the counterpart with $K_{\mathrm{s}}=18.05$. We observed this source in quiescence in both $J$ and $K_{s}$ bands (see Table 3 ). Our observation in the $K_{s}$ band yielded a non-detection down to a limiting magnitude of $K_{s}>17.9$, whereas in the $J$ band we just detect a faint candidate counterpart with $J=21.0 \pm 0.3$ (see Fig. A1a).

\subsubsection{Swift J174510.8-262411}

This XRT went into outburst on 2012 September 16 (Cummings et al. 2012) and was observed with GROND $1 \mathrm{~d}$ after (Rau et al. 2012b), where they found a candidate counterpart with an apparent magnitude of $i^{\prime} \sim 17.8$ and $J \sim 16.5 \pm 0.5$, which was then spectroscopically confirmed by de Ugarte Postigo et al. (2012). Subsequent optical observations were performed by Hynes et al. (2012) and Russell et al. (2012); the former did not detect the counterpart, whereas the latter detected the source previously identified by Rau et al. (2012b), this time with a magnitude of $i^{\prime}=17.59 \pm 0.07$. We observed this source $5 \mathrm{yr}$ after its outburst and detect the counterpart in the NIR (see Fig. A1b) with a magnitude of $K_{\mathrm{s}}=18.63 \pm 0.15$ (see Table 3) with a position consistent with that reported in Rau et al. (2012b). Muñoz-Darias et al. (2013) estimated a distance of $d>7 \mathrm{kpc}$ (see Table 1) and with $N_{\mathrm{H}}=1.70 \pm 0.04 \times 10^{22}$ $\mathrm{cm}^{-2}$ (Tomsick, DelSanto \& Belloni 2012), we get an extinction of $A_{K_{\mathrm{s}}}=0.918$, which we use to estimate a limiting absolute magnitude of $M_{K}>3.4$. This would make the donor star a K0V star or later (Tokunaga 2000; Drilling \& Landolt 2000), consistent with the hydrogen emission lines detected by de Ugarte Postigo et al. (2012).

\subsubsection{XTE J1752-223}

This source was discovered on 2009 October 23 (Markwardt et al. 2009) and later confirmed to be a strong accreting BH candidate (Nakahira et al. 2010; Muñoz-Darias et al. 2010; Shaposhnikov et al. 2010; Curran et al. 2011). An optical counterpart was identified by Torres et al. (2009b), later confirmed by optical spectra and an NIR detection (Torres et al. 2009c) with a magnitude of $K_{\mathrm{s}}=$ $15.83 \pm 0.01$. Approximately 9 months after the outburst, Russell et al. (2010) reported optical and NIR quiescent magnitudes for the counterpart of $V=21.2 \pm 0.3$ and $K_{\mathrm{s}}=17.1 \pm 0.1$, respectively. However, Ratti et al. (2012) showed that the source had not yet reached the quiescent state at the point in time related to the observations of Russell et al. (2010), as they did not detect the source 10 months later down to a limiting magnitude of $i^{\prime}>24.77$, making the difference between the source brightness in outburst and in quiescence at least 8 mag. We obtained NIR observations of this source $6 \mathrm{yr}$ after its outburst and did not detect any source at the position of the outburst counterpart, down to a limiting magnitude of $K_{\mathrm{s}}>$ 19.6. This is 2.5 mag fainter than reported before by Russell et al. (2010). With a distance of $d=6 \pm 2 \mathrm{kpc}$, derived by Ratti et al. (2012) (see Table 1), and using $N_{\mathrm{H}}=4.5 \times 10^{21} \mathrm{~cm}^{-2}$ (Dickey \& Lockman 1990), we get an extinction of $A_{K_{\mathrm{s}}}=0.243$, which results in a limiting absolute magnitude of $M_{K}>5.3$, which means that the donor star is a main-sequence star with spectral type M0 or later (Drilling \& Landolt 2000; Tokunaga 2000). This is consistent with the constraints on the spectral type given in Ratti et al. (2012) and with the hydrogen emission lines found in the spectra by Torres et al. $(2009 \mathrm{c})$.

\subsubsection{MAXI J1807 + 132}

MAXI J1807+132 was discovered on 2017 March 13 by MAXI/GSC (Negoro et al. 2017). A UV/optical counterpart was detected soon after by Kennea et al. (2017b) and Denisenko (2017), who reported magnitudes of $B=18.3 \pm 0.2$ and $V=17.82$, respectively. Furthermore, Denisenko (2017) reported the quiescent magnitude of this optical counterpart as $r^{\prime}=21.19 \pm 0.09$ and $i^{\prime}=$ $21.38 \pm 0.04$ from PanSTARRS-1 archival images. Spectroscopy taken with GTC/OSIRIS revealed spectral features consistent with that of an LMXB (Munoz-Darias et al. 2017; Shidatsu et al. 2017b). Tachibana et al. (2017) detected in the optical a decay in magnitude of $\sim 0.4 \mathrm{mag} \mathrm{d}^{-1}$, which coincided with a decay in the X-ray 
Table 3. Outburst and quiescent magnitudes.

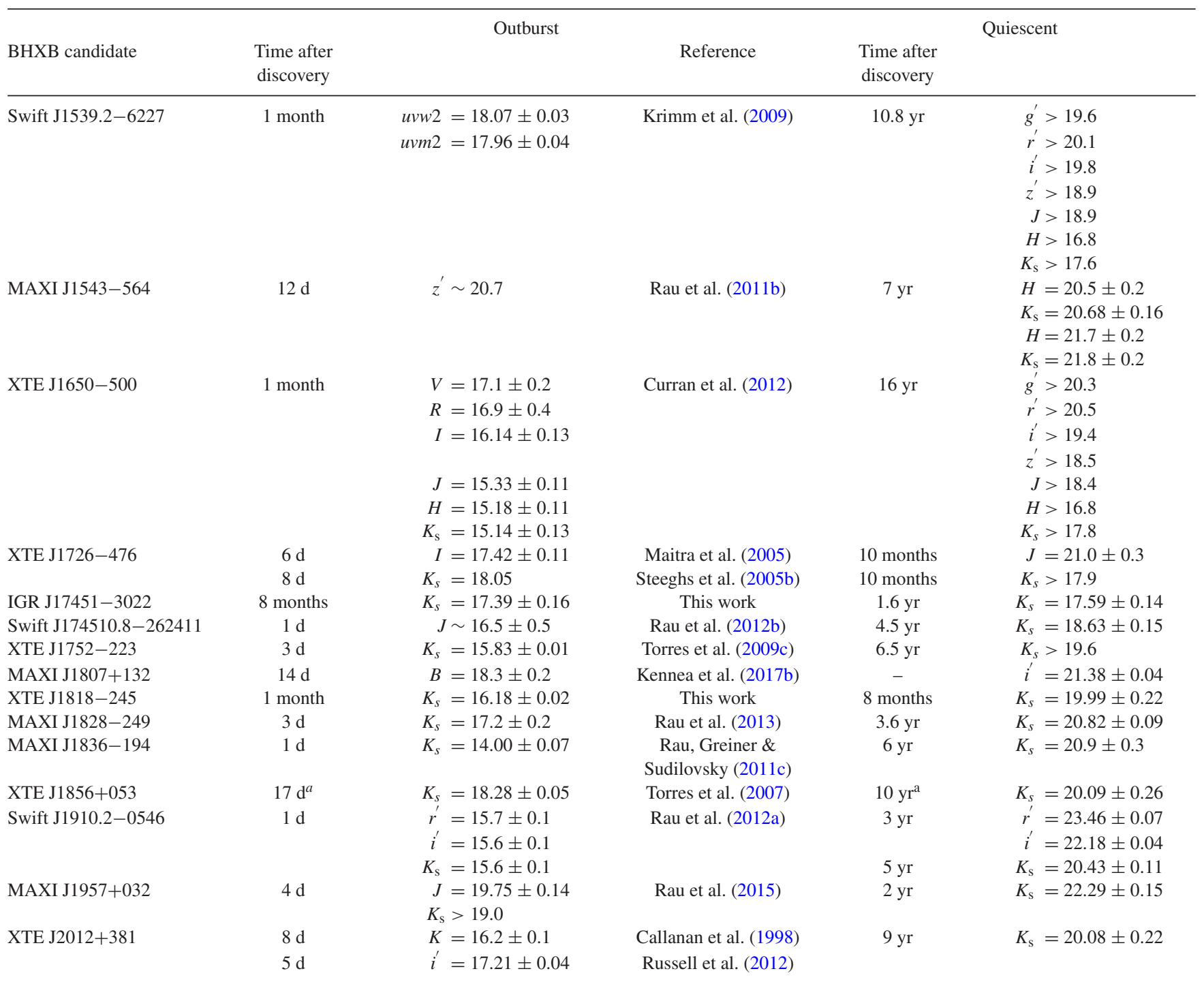

Note. ${ }^{a}$ Time after the second outburst was detected. All the values in quiescence are calculated in this paper, with the exception of the magnitude for MAXI $\mathrm{J} 1807+132$, which is from the PanSTARRS 1 catalogue. The magnitudes are in the AB system, unless indicated otherwise (conversion between the Vega and $\mathrm{AB}$ systems was done following Blanton \& Roweis 2007, i.e. $\left.J_{\mathrm{AB}}=J_{\mathrm{VEGA}}+0.91, H_{\mathrm{AB}}=H_{\mathrm{VEGA}}+1.39, K_{\mathrm{SAB}}=K_{\mathrm{sVEGA}}+1.85\right)$.

(Armas Padilla et al. 2017). Furthermore, after the X-ray fading phase of MAXI J1807+132, the optical counterpart varied its magnitude from $r^{\prime}=20.76 \pm 0.07$ to $r^{\prime}=19.34 \pm 0.02$ in a 5 -d interval (Kong et al. 2017). We observed this X-ray source in the $i$ band (see Fig. A1c) 4 months after its outburst started, and detected the optical counterpart reported by Kennea et al. (2017b) with an magnitude of $i^{\prime}=20.26 \pm 0.05$. This is 2.3 mag brighter than the archival magnitude from PanSTARRS-1, which implies that the source had not fully returned to quiescence yet at the time of our observations.

\subsubsection{MAXI J1828-249}

Nakahira et al. (2013) discovered MAXI J1828-249 in a soft state on 2013 October 15. An uncatalogued UV point source was detected with Swift as a possible counterpart, with a magnitude of $u v m 2=18.64 \pm 0.04 \pm 0.03$ (with 0.04 and 0.03 being statistical and systematic uncertainties, respectively, Kennea et al. 2013). Follow-up observations by Rau, Tanga \& Greiner (2013) detected a counterpart at $H=16.9 \pm 0.1$ and $K_{\mathrm{s}}=17.2 \pm 0.2$. A month later, another NIR observation (D' Avanzo et al. 2013) revealed fading of this counterpart, with a magnitude of $H=18.7 \pm 0.1$, which implies that this source is the counterpart. We detect this source in our NIR observations with $K_{\mathrm{s}}=20.82 \pm 0.09$ (see Table 3), 3.6 mag fainter than in outburst (see Fig. A1d).

\subsubsection{MAXI J1836-194}

MAXI J1836-194 was first detected on 2011 August 29 by MAXI/GSC and Swift/BAT, as a hard X-ray transient (Negoro et al. 2011b). Swift observations revealed an optical counterpart with a magnitude of $V=16.22 \pm 0.04$ (Kennea et al. 2011b). GROND observations detected the optical counterpart with magnitudes of $g^{\prime}=16.21 \pm 0.05, r^{\prime}=15.92 \pm 0.05, i^{\prime}=15.53 \pm 0.01, z^{\prime}=$ $15.09 \pm 0.05, J=14.77 \pm 0.05, H=14.34 \pm 0.05$, and $K_{\mathrm{s}}=$ $14.00 \pm 0.07 \mathrm{mag}$ (Rau et al. 2011c). This XRB has a face-on accretion disc with an inclination between $4^{\circ}$ and $15^{\circ}$, and a lower 


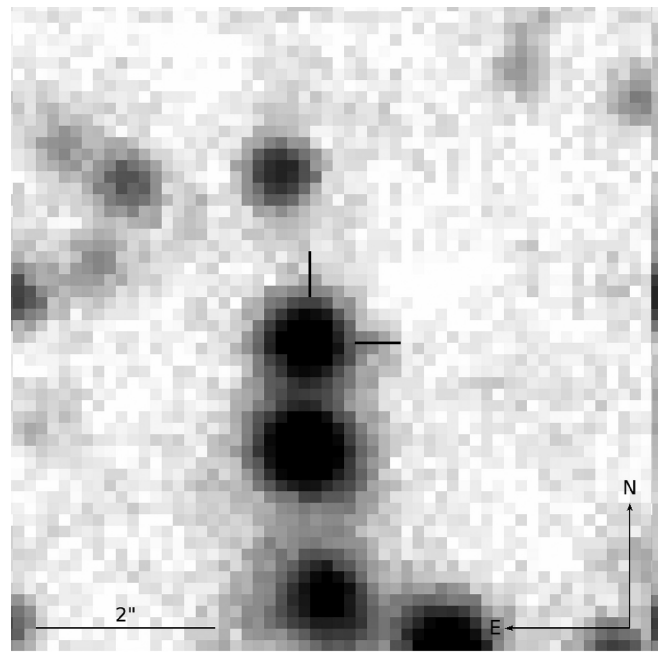

(a)

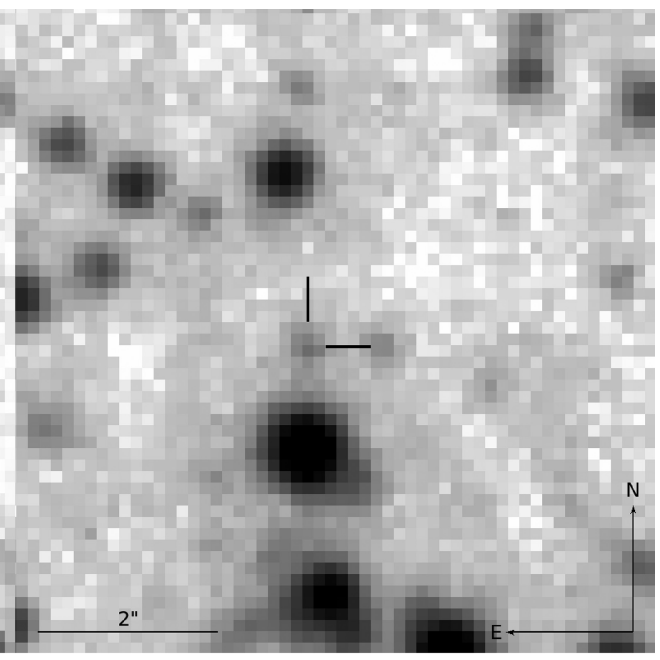

(b)

Figure 3. PANIC $K_{\mathrm{S}}$-band image of XTE J1818-245 in (a) outburst and (b) quiescence.

limit on the compact object mass of $1.9 \mathrm{M}_{\odot}$ (Russell et al. 2014). Six years after the first discovery outburst, we just detect the NIR counterpart at the position given by Kennea et al. (2011b), with a magnitude of $K_{\mathrm{s}}=20.9 \pm 0.3$ (see Fig. A1e). This source has an estimated distance of $d=7 \pm 3 \mathrm{kpc}$ (Russell et al. 2014, see Table 1) and an $N_{\mathrm{H}}=2.0 \pm 0.4 \times 10^{21} \mathrm{~cm}^{-2}$ (Kennea et al. 2011b), which results in an extinction of $A_{K_{\mathrm{s}}}=0.108$ and an absolute magnitude of $M_{K}>6.5$. This would make the donor star a M2 main-sequence star or later (Drilling \& Landolt 2000; Tokunaga 2000).

\subsubsection{XTE J1856+053}

This BHXB candidate was discovered on 1996 September 17 (Marshall et al. 1996), with a new outburst observed 9 yr later on 2007 February 28 (Levine \& Remillard 2007). It seems that the source was already in outburst around 2007 February 22 (Krimm et al. 2007). Days after the 2007 outburst detection, NIR observations were performed and yielded a counterpart with an apparent magnitude of $K_{\mathrm{s}}=18.28 \pm 0.05$ (Torres et al. 2007) that had faded 2 months after the outburst to $K_{\mathrm{s}}=19.7 \pm 0.1$. In 2015, a new outburst from XTE J1856+053 was detected by MAXI/GSC (Suzuki et al. 2015; Negoro et al. 2015b) and Swift/XRT (Sanna et al. 2015). Two years later, we observed this source in the NIR (see Fig. A1f). We detect the same counterpart reported by Torres et al. (2007), this time with a magnitude of $K_{s}=20.09 \pm 0.26$, which is consistent at the $1 \sigma$ level with the quiescent value reported in 2007 (see Table 4).

\subsubsection{Swift J1910.2-0546}

An outburst from Swift 1910.2-0546 was discovered on 2012 May 31 (Usui et al. 2012). Optical/NIR observations made $1 \mathrm{~d}$ after by Rau et al. (2012a) revealed a counterpart with $g^{\prime}=16.1 \pm 0.1, r^{\prime}=$ $15.7 \pm 0.1, i^{\prime}=15.6 \pm 0.1, z^{\prime}=15.3 \pm 0.1, J=15.5 \pm 0.1, H=$ $15.5 \pm 0.1$, and $K_{\mathrm{s}}=15.6 \pm 0.1 \mathrm{mag}$. That same day, Cenko \& Ofek (2012) also detected this counterpart at $R=16.11$. The optical spectra reported by Charles, Cornelisse \& Casares (2012) are typical of an LMXB. In our $K_{\mathrm{s}}$-band observations (see Fig. A1g), we detect the quiescent counterpart with an apparent magnitude of
$K_{\mathrm{s}}=20.43 \pm 0.11$, while in our optical observations, the counterpart is detected with an apparent magnitude of $r^{\prime}=23.46 \pm 0.07$ and $i^{\prime}=22.18 \pm 0.04$.

\subsubsection{MAXI J1957+032}

Discovered on 2015 May 11 (Cherepashchuk et al. 2015; Negoro et al. 2015a), MAXI J1957+032 was classified as an LMXB (Munoz-Darias et al. 2017). Subsequent optical and NIR observations revealed a candidate counterpart with magnitudes $r^{\prime}=$ $20.03 \pm 0.14$ and $J=19.75 \pm 0.14$ (Rau et al. 2015). In the $K_{\mathrm{s}}$ band there was no detection, down to a limiting magnitude of $K_{\mathrm{s}}>19.0$. A day later the source had faded to $r^{\prime}=21.36 \pm 0.14$, and since this quick decay was similar to the one observed in X-rays (Molkov et al. 2015), it is most likely that the NIR source is the counterpart. This source seemed to have brightened approximately 1 mag when observed in the optical some months later (Guver et al. 2015), coinciding with a new outburst from MAXI J1957+032 (Sugimoto et al. 2015). Optical spectra were taken (Buckley et al. 2016), which turned out to be featureless. This is consistent with a highinclination compact system, where the optical spectra have almost undetectable emission lines (Baglio et al. 2016). MAXI J1957+032 shows short duration outbursts $(<5 \mathrm{~d})$ and it has a high recurrence rate (4 in 16 months). Mata Sánchez et al. (2017) found not only that the optical spectra is consistent with MAXI J1957+032 being a short-period LMXB but also that the short duration and short recurrence time of the outburst are reminiscent of an accreting millisecond X-ray pulsar. We detect an NIR source (see Fig. A1h), at the position indicated by Rau et al. (2015), with $K_{s}=22.29 \pm 0.15$ mag. Using the distance estimate of $d \sim 6 \mathrm{kpc}$ and the column density $N_{\mathrm{H}}=1.7 \times 10^{21} \mathrm{~cm}^{-2}$ from Mata Sánchez et al. (2017) (see Table 1), we get an extinction of $A_{K_{s}}=0.091$, and thus, an absolute magnitude of $M_{K}>8.3$, making it consistent with an M6 main-sequence star or later (Tokunaga 2000; Drilling \& Landolt 2000).

\subsubsection{XTE J2012+381}

This source went into outburst on 1998 May 24 (Remillard et al. 1998). A radio source was identified as a possible counterpart 
Table 4. Magnitudes previously reported as quiescent compared to our more recent (and fainter) values.

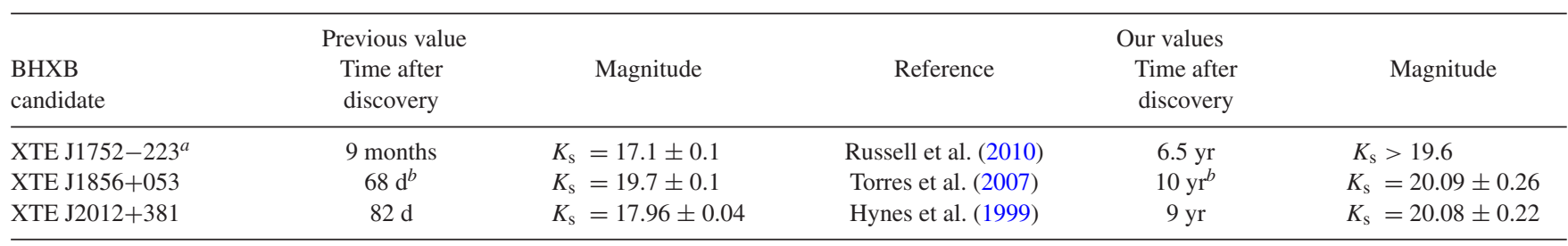

Notes. ${ }^{a}$ Ratti et al. (2012) showed that the source had not yet reached the quiescent state in the $i$ band at the time of the Russell et al. (2010) observations. We here report the first measurement in the $K_{\mathrm{S}}$ band. ${ }^{\mathrm{b}}$ Time after the second outburst was detected.

(Hjellming, Rupen \& Mioduszewski 2000), along with an optical source $(V=17.22 \pm 0.2 \mathrm{mag}$, Hynes et al. 1998) and an NIR source ( $K=16.2 \pm 0.1 \mathrm{mag}$, Callanan et al. 1998). Subsequent optical and NIR observations (done on 1998 August 14) revealed that the NIR source faded by $1.8 \mathrm{mag}$, from $K=16.2 \pm 0.1$ to $K_{s}=17.96 \pm 0.04$ (Hynes et al. 1999) in $71 \mathrm{~d}$. Moreover, this source was identified as the optical counterpart to the radio source identified by Hjellming et al. (2000). In our NIR observations (see Fig. A1i), performed $8 \mathrm{yr}$ after the outburst, we detect the same source, with a magnitude of $K_{s}=20.08 \pm 0.22$. We report this as the quiescent magnitude of the counterpart (see Table 4).

\section{DISCUSSION}

Shahbaz \& Kuulkers (1998) derived an empirical relation between $\Delta V$ and $P_{\text {orb }}$ for $8 \mathrm{BH}$ XRTs, where $P_{\text {orb }}<1 \mathrm{~d}$. The relation is an inverse linear correlation, which they suggest is explained by the fact that at longer $P_{\text {orb }}$, the secondary star is larger and hence, more luminous than a system with shorter $P_{\text {orb }}$, making the outburst amplitude smaller. As already mentioned in Miller-Jones et al. (2011b) and Revnivtsev, Zolotukhin \& Meshcheryakov (2012), the inclination $i$ is an important parameter as well. We searched the literature and found $i$ for 6 of these 8 sources (see Table A1) and plot $\Delta V$ versus $i$ and $P_{\text {orb }}$ in Fig. 4(a). We also include other sources for which $\Delta V, i$, and $P_{\text {orb }}$ have become available since the work of Shahbaz \& Kuulkers (1998) (see Table A1). Since many of the sources analysed are known to have had multiple outbursts (e.g. XTE J1550-564, GX 339-4, GS 2023+338), we take the brightest outburst magnitude and the faintest quiescent magnitude reported. The latter is because there are sources like A 0620-00 whose mean brightness varies even while in quiescence ('active' and 'passive' states; Cantrell et al. 2008). However, note that the outburst amplitudes are probably lower limits, to account for the fact that one may have missed the peak magnitude in the sparsely sampled outbursts and that there could be a brightness variation in quiescence.

For 10 of the 15 sources that we observed we have outburst and quiescent magnitudes in the $K_{\mathrm{s}}$ band (see Table 3 ), allowing us to derive a lower limit on the outburst amplitude $\Delta K_{\mathrm{s}}$. As with the optical data, we complement our data with that available in the literature. We plot $\Delta K_{\mathrm{S}}$ versus $i$ and $P_{\text {orb }}$ and show the results in Fig. 4(b). We performed a 3D least squares plane fit (see Fig. A2) for both data sets and obtained

$\Delta V=(-2.63 \pm 1.01) \log P_{\text {orb }}+(0.03 \pm 0.03) i+(7.82 \pm 1.94)$

with $\chi^{2}=16.73$ and residuals plotted in Fig. A2(c), and

$\Delta K_{\mathrm{S}}=(-1.32 \pm 0.99) \log P_{\mathrm{orb}}-(0.04 \pm 0.03) i+(6.89 \pm 2.16)$ with $\chi^{2}=2.81$ and residuals plotted in Fig. A2(d). Taking into account the $\chi^{2}$ values ${ }^{5}$ and the residuals, we see that the fit is not adequate for either data set. The Pearson correlation coefficient between $\Delta V$ and $\log P_{\text {orb }}$ is $r=-0.56$ and the coefficient between $\Delta V$ and $i$ is $r=0.03$, whereas the coefficient between $\Delta K_{\mathrm{s}}$ and $\log P_{\text {orb }}$ is $r=-0.45$ and the coefficient between $\Delta K_{\mathrm{s}}$ and $i$ is $r=$ -0.46 . Based on these coefficients, we conclude that here is no strong correlation between the variables.

To explore these results further, we derive the approximate size of the projected area of the accretion disc of a system with a given mass ratio $q, i, P_{\text {orb }}$ and a mass $M_{1}$ for the compact object. From geometrical arguments, we know that the projected area $A_{\mathrm{p}}$ of the disc can be approximated by $A_{p}=\pi\left(R_{\text {acc }} \cos i\right)^{2}$ where $R_{\text {acc }}$ is the accretion disc radius. Following Frank, King \& Raine (2002), we derive $R_{\text {acc }}$ as

$R_{\mathrm{acc}}=\frac{1}{2} P_{\mathrm{orb}}^{\frac{2}{3}}(1+q)^{\frac{4}{3}}\left(\frac{M_{1}}{\mathrm{M}_{\odot}}\right)^{\frac{1}{3}}(0.500-0.227 \log q)^{4} \mathrm{R}_{\odot}$,

where $P_{\text {orb }}$ is in hours. Clearly, $A_{p}$ increases with $P_{\text {orb }}$ and decreases with $i$. It was shown and parametrized by van Paradijs \& McClintock (1994) that, besides $P_{\text {orb }}$ and $i, L_{X}$ is also important for the absolute magnitude of the disc in outburst. They found that the absolute magnitude during outburst $M_{V, O}$ depends on $P_{\text {orb }}$ and the outburst X-ray luminosity $L_{X, O}$, as $M_{V, O} \propto \log \left(P_{\text {orb }}^{2 / 3} L_{X, O}^{1 / 2}\right)$. The outburst Xray luminosity also depends on $P_{\text {orb }}\left(L_{X, O} \propto P_{\text {orb }}^{0.64}\right.$, Wu et al. 2010).

Another factor is that the quiescent $\mathrm{X}$-ray luminosity shows a dependence on $P_{\text {orb }}$ as well (i.e. Lasota 2000; Garcia et al. 2001; Jonker et al. 2007; Lasota 2008; Degenaar et al. 2009; Rea et al. 2011; Jonker et al. 2012) of the form $L_{X, Q} \propto P_{\text {orb }}^{1.77}$. Moreover, the quiescent magnitude $M_{V, Q}$ might be the result of (a) the contribution from the light of disc, which, like in outburst, can in principle be due to reprocessing of the X-ray light on its surface (van Paradijs \& McClintock 1994), but also due to energy generation on the disc itself, although we ignore this below; and (b) the light from the donor star $M_{V \text {, donor }}$, which in outburst is difficult to detect. In some cases, the donor star is not even detected in the quiescent state (e.g. Torres et al. 2015). Thus, $M_{V, Q} \propto \log \left(P_{\mathrm{orb}}^{2 / 3} L_{X, Q}^{1 / 2}\right)+M_{V \text {, donor }}$. If we ignore irradiation of the donor star in both outburst and quiescence, filling in all the dependences and relations given above, we derive $\Delta V=$ $M_{V, Q}-M_{V, O} \propto \log P_{\mathrm{orb}}^{0.565}$ (we plot this correlation as a dash line in Fig. 4). Therefore, for a given $i$, the same $L_{X, O}$, and $q$, systems would be expected to have slightly larger outburst amplitudes for longer $P_{\text {orb }}$, counter to the relation reported in Shahbaz \& Kuulkers (1998). A factor not included above is that in the NIR, emission from a non-thermal component, like a jet (e.g. Corbel \& Fender

${ }^{5}$ To calculate $\chi^{2}$ we assigned to the data points without reported uncertainties, the mean uncertainty of $P_{\text {orb }}, i, \Delta V$, or $\Delta K_{s}$ within their respective data sets. 


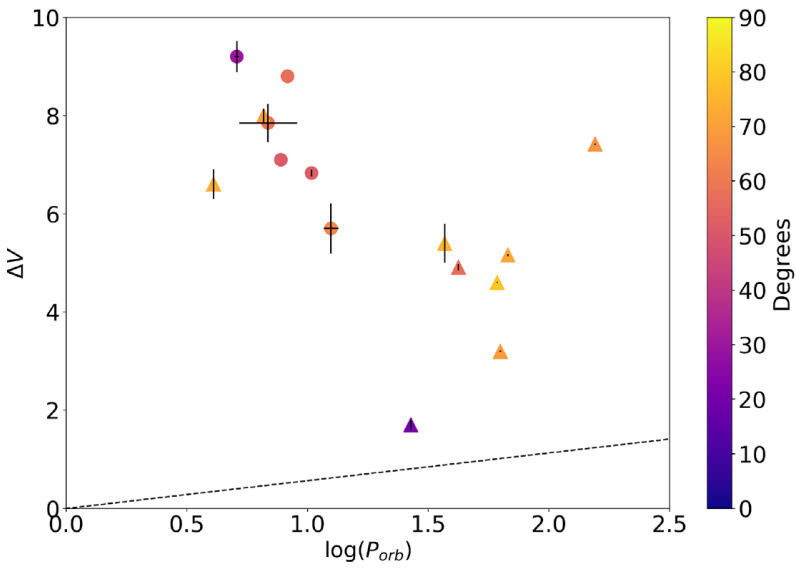

(a)

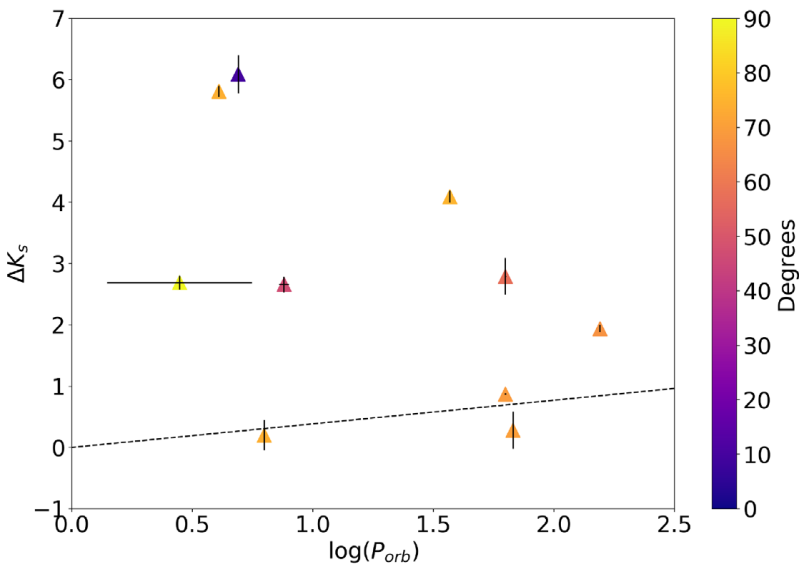

(b)

Figure 4. (a) Plot of the $V$-band outburst amplitude versus the logarithm of the orbital period $P_{\text {orb }}$ and the inclination angle $i$, where the latter is indicated with different colours (for references see Table A1); adapted from Shahbaz \& Kuulkers (1998). The sources for which Shahbaz \& Kuulkers (1998) found a relation are indicated in round symbols, while the sources taken from other publications are indicated in triangular symbols. (b) Same as for panel (a) but now for the $K_{s}$-band amplitude. The dashed line indicates the relations $\Delta V \propto \log P_{\text {orb }}^{0.565}$. No error bars are shown if the original work did not provide uncertainties to their estimates.

2002; Russell et al. 2006; Russell, Fender \& Jonker 2007; Russell \& Fender 2008), possibly contributes to the observed NIR light.

In order to try and assess the influence that the low number of sources involved in the work of Shahbaz \& Kuulkers (1998) (and also to a lesser extent in the work presented here), we determine the probability of randomly selecting 8 systems and finding a correlation between $\Delta V$ and $P_{\text {orb }}$. We find that it ranges between 17.5 per cent and 30.5 per cent (based on selecting 1000 different samples of 8 elements from a simulation of 100 systems with a flat distribution between $0<P_{\text {orb }}<12$ and $0<\Delta V<10$ ). We conclude that the correlation reported by Shahbaz \& Kuulkers (1998) was probably caused by small number statistics.

We do note that some of the observational correlations used above to determine the expected relation between $P_{\text {orb }}, i$, and $\Delta V\left(\Delta K_{s}\right)$ may suffer from low number statistics themselves implying that the predicted correlation between $\Delta V$ and $P_{\text {orb }}$ itself is uncertain.

\section{CONCLUSIONS}

We observed 15 BHXB candidates in quiescence in the NIR and optical in order to investigate which of these sources would be promising targets for $\mathrm{BH}$ mass determinations. We detected 2 with $K_{\mathrm{s}}<20$ (AB magnitude) out of our sample, which depending on their $P_{\text {orb }}$, may allow for time-resolved spectroscopic observations to be taken with 8-10 m class telescopes. Such measurements for fainter systems will have to await the arrival of larger or space-based telescopes. Of our 15 observed sources, XTE J1818-245 and IGR J17451-3022 were observed in quiescence and in outburst, while the other 13 were observed only in quiescence. XTE J1818-245 presents an outburst-quiescence magnitude difference of about $\sim 4$ mag in the $K_{\mathrm{s}}$ band. IGR J17451-3022 was analysed with differential photometry, as it was not clear which, if any, of the two sources inside the 99.7 per cent confidence radius around it is the counterpart. One source has a magnitude change consistent in direction with the fading in the X-rays. However, the outburst-quiescence magnitude difference is small. It is conceivable that this is due to the fact that the system has a high inclination, so the projected surface area of the disc is small. Out of the 13 sources observed only in quiescence, we detect NIR and optical counterparts for ten of them, while for the remaining three sources we report the limiting magnitudes. Of the ten sources with detected quiescent counterparts, we present measurements for the first time for five of them, and in contrast, two of them had previously published quiescent magnitudes that we update now with fainter magnitudes, showing that these BHXBs had not yet reached their quiescent states at the epoch of the observations reported in the literature before. We analysed the $K_{\mathrm{s}}$-band and $V$-band outburst amplitudes with respect to $P_{\text {orb }}$ and $i$ of the system for sources in our sample and for BHXBs for which we could find published values of $\Delta K_{s}, \Delta V, P_{\mathrm{orb}}$, and $i$. We find best fits to the data although the fits are not formally acceptable, showing that probably additional parameters to $P_{\text {orb }}$ and $i$ are important (e.g. emission from a jet, energy generation on the disc, light contribution from the donor star). Using theoretical arguments and observed relations, we further derive a correlation between $\Delta V$ and $P_{\text {orb }}$ of $\Delta V \propto \log P_{\text {orb }}^{0.565}$; however, such a correlation also provides no good fit to the data, probably due to the observed relations being affected by low number statistics, which could make our derived correlation less certain, and reinforcing the idea that additional parameters to $P_{\text {orb }}$ and $i$ should be considered.

\section{ACKNOWLEDGEMENTS}

This study is based on observations made with ESO Telescopes at the La Silla Paranal Observatory under programmes 099.A-9025(A) and 1101.D-0105(A). PGJ and KML acknowledge funding from the European Research Council under ERC Consolidator Grant agreement no 647208. Part of the funding for GROND (both hardware as well as personnel) was generously granted from the Deutsche Forschungsgemeinschaft Gottfried Wilhelm Leibniz Prize to Prof. G. Hasinger (DFG grant HA 1850/28-1). MAPT acknowledges support via a Ramón y Cajal Fellowship (RYC-2015-17854). MAPT also acknowledges support by the Spanish Ministry of Economy, Industry and Competitiveness under grant AYA2017-83216-P. This research is based on observations made with the William Herschel Telescope operated on the island of La Palma by the Isaac Newton Group in the Spanish Observatorio del Roque de los Muchachos of the Instituto de Astrofísica de Canarias; with the Keck Telescope at the W. M. Keck Observatory, which is operated as a scientific 
partnership among the California Institute of Technology, the University of California and the National Aeronautics and Space Administration. The Observatory was made possible by the generous financial support of the W. M. Keck Foundation. The authors wish to recognize and acknowledge the very significant cultural role and reverence that the summit of Maunakea has always had within the indigenous Hawaiian community. We are most fortunate to have the opportunity to conduct observations from this mountain. We have made use of the SIMBAD data base, operated at CDS, Strasbourg, France, of the NASA/IPAC Extragalactic Database (NED) which is operated by the Jet Propulsion Laboratory, California Institute of Technology, under contract with the National Aeronautics and Space Administration.

\section{REFERENCES}

Abbott B. P. et al., 2016a, Phys. Rev. Lett., 116, 061102

Abbott B. P. et al., 2016b, Phys. Rev. Lett., 116, 241103

Armas Padilla M., Wijnands R., Degenaar N., Munoz-Darias T., JimenezIbarra F., Mata Sanchez D., Casares J., Charles P. A., 2017, Astron. Telegram, 1022

Augusteijn T., 1992, An Introduction to Astronomical Photometry. Astronomical Institute 'Anton Pannekoek', University of Amsterdam, the Netherlands

Baglio M. C., D’Avanzo P., Campana S., Goldoni P., Masetti N., MuñozDarias T., Patiño-Álvarez V., Chavushyan V., 2016, A\&A, 587, A102

Bahramian A., Heinke C. O., Altamirano D., Wijnands R., 2015, Astron. Telegram, 7570

Beer M. E., Podsiadlowski P., 2002, MNRAS, 331, 351

Belczynski K., Wiktorowicz G., Fryer C. L., Holz D. E., Kalogera V., 2012, ApJ, 757, 91

Belloni T. M., Motta S. E., Muñoz-Darias T., 2011, Bull. Astron. Soc. India, 39, 409

Bernardini F., Russell D. M., Kolojonen K. I. I., Stella L., Hynes R. I., Corbel S., 2016, ApJ, 826, 149

Bertin E., 2006, in Gabriel C., Arviset C., Ponz D., Enrique S., eds, ASP Conf. Ser. Vol. 351, Astronomical Data Analysis Software and Systems XV. Astron. Soc. Pac., San Francisco, p. 112

Bertin E., Arnouts S., 1996, A\&AS, 117, 393

Bertin E., Mellier Y., Radovich M., Missonnier G., Didelon P., Morin B., 2002, in Bohlender D. A., Durand D., Handley T. H., eds, ASP Conf. Ser. Vol. 281, Astronomical Data Analysis Software and Systems XI. Astron. Soc. Pac., San Francisco, p. 228

Blanton M. R., Roweis S., 2007, AJ, 133, 734

Blissett R. J., Pedersen H., Veron M., Veron P., Schuster H.-E., 1983, IAU Circ., 3858

Bohlin R. C., Savage B. D., Drake J. F., 1978, ApJ, 224, 132

Bradt H. V., Rothschild R. E., Swank J. H., 1993, A\&AS, 97, 355

Buckley D. A. H., Kotze M. M., Charles P. A., Sanchez D. M., Munoz-Darias T., Israel G., Masetti E. J. M. N., Jonker P., 2016, Astron. Telegram, 9649

Buxton M., Bailyn C., Maitra D., 2005, Astron. Telegram, 418

Buxton M. M., Bailyn C. D., Capelo H. L., Chatterjee R., Dinçer T., Kalemci E., Tomsick J. A., 2012, AJ, 143, 130

Cadolle Bel M. et al., 2009, A\&A, 501, 1

Callanan P., McCarthy J., Garcia M., McClintock J., 1998, IAU Circ., 6933

Cantrell A. G., Bailyn C. D., McClintock J. E., Orosz J. A., 2008, ApJ, 673, L159

Casares J., Jonker P. G., 2014, Space Sci. Rev., 183, 223

Casares J., Charles P. A., Naylor T., 1992, Nature, 355, 614

Casares J. et al., 2009, ApJS, 181, 238

Casares J., Jonker P. G., Israelian G., 2017, in Alsabti A., Murdin P., eds, X-Ray Binaries, Handbook of Supernovae. Springer-Verlag, Berlin

Castro-Tirado A. J., Kilmartin P., Gilmore A., Petterson O., Bond I., Yock P., Sanchez-Fernandez C., 2001, IAU Circ., 7707

Cenko S. B., Ofek E. O., 2012, Astron. Telegram, 4146

Chakrabarty D., Jonker P. G., Markwardt C. B., 2011, Astron. Telegram, 3407
Chakrabarty D., Jonker P. G., Markwardt C. B., 2014, Astron. Telegram, 6533

Chambers K. C. et al., 2016, preprint (arXiv:1612.05560)

Charles P., Cornelisse R., Casares J., 2012, Astron. Telegram, 4210

Charles P. A., Coe M. J., 2003, preprint (arXiv:astro-ph/0308020)

Chaty S., Mirabel I. F., Goldoni P., Mereghetti S., Duc P.-A., Martí J., Mignani R. P., 2002, MNRAS, 331, 1065

Chaty S., Charles P. A., Martí J., Mirabel I. F., Rodríguez L. F., Shahbaz T., 2003, MNRAS, 343, 169

Chenevez J., Vandbaek Kroer L., Budtz-Jorgensen C., Brandt S., Lund N., Westergaard N. J., Kuulkers E., Wilms J., 2014, Astron. Telegram, 6451

Cherepashchuk A. M., Molkov S. V., Lutovinov A. A., Postnov K. A., 2015, Astron. Telegram, 7506

Corbel S., Fender R. P., 2002, ApJ, 573, L35

Corral-Santana J. M., Casares J., Shahbaz T., Zurita C., Martínez-Pais I. G., Rodríguez-Gil P., 2011, MNRAS, 413, L15

Corral-Santana J. M., Casares J., Shahbaz T., Zurita C., Martínez-Pais I. G., Rodríguez-Gil P., 2013a, Revista Mexicana de Astronomia y Astrofisica Conference Series, 42, 3

Corral-Santana J. M., Casares J., Muñoz-Darias T., Rodríguez-Gil P., Shahbaz T., Torres M. A. P., Zurita C., Tyndall A. A., 2013b, Science, 339, 1048

Corral-Santana J. M., Casares J., Muñoz-Darias T., Bauer F. E., MartínezPais I. G., Russell D. M., 2016, A\&A, 587, A61

Cummings J. R. et al., 2012, GRB Coordinates Network, 13775

Curran P. A., Chaty S., 2013, A\&A, 557, A45

Curran P. A., Maccarone T. J., Casella P., Evans P. A., Landsman W., Krimm H. A., Brocksopp C., Still M., 2011, MNRAS, 410, 541

Curran P. A., Chaty S., Zurita Heras J. A., 2012, A\&A, 547, A41

D’Avanzo P., Campana S., Covino S., Goldoni P., Munoz-Darias T., Masetti N., Palazzi E., 2013, Astron. Telegram, 5559

de Ugarte Postigo A., Sanchez-Ramirez R., Munoz-Darias T., Gorosabel J., Thoene C. C., Cabrera-Lavers A., 2012, Astron. Telegram, 4388

Degenaar N. et al., 2009, MNRAS, 396, L26

Denisenko D., 2017, Astron. Telegram, 1021

Dickey J. M., Lockman F. J., 1990, ARA\&A, 28, 215

Drilling J. S., Landolt A. U., 2000, in Cox A. N., ed., Allen's Astrophysical. Quantities, 4th ednNormal Stars. Springer-Verlag, New York, p. 381

Dubus G., Hameury J.-M., Lasota J.-P., 2001, A\&A, 373, 251

Farr W. M., Sravan N., Cantrell A., Kreidberg L., Bailyn C. D., Mandel I., Kalogera V., 2011, ApJ, 741, 103

Fender R. P., Kuulkers E., 2001, MNRAS, 324, 923

Fender R. P., Gallo E., Jonker P. G., 2003, MNRAS, 343, L99

Fitzpatrick E. L., 1999, PASP, 111, 63

Flewelling H. A. et al., 2016, preprint (arXiv:1612.05243)

Frank J., King A., Raine D. J., 2002, Accretion Power in Astrophysics, 3rd edn. Cambridge Univ. Press, New York

Fryer C. L., Kalogera V., 2001, ApJ, 554, 548

Fryer C. L., Belczynski K., Wiktorowicz G., Dominik M., Kalogera V., Holz D. E., 2012, ApJ, 749, 91

Gaia Collaboration et al., 2016, A\&A, 595, A1

Garcia M. R., Wilkes B. J., 2002, Astron. Telegram, 104

Garcia M. R., McClintock J. E., Narayan R., Callanan P. J., 1998, in Howell S., Kuulkers E., Woodward C., eds, ASP Conf. Ser. Vol. 137, Wild Stars in the Old West. Astron. Soc. Pac., San Francisco,p. 506

Garcia M. R., McClintock J. E., Narayan R., Callanan P., Barret D., Murray S. S., 2001, ApJ, 553, L47

Garner A. et al., 2015, Astron. Telegram, 7663

Gelino D. M., Harrison T. E., McNamara B. J., 2001, AJ, 122, 971

Gelino D. M., Balman Ş., Kızıloğlu Ü., Yılmaz A., Kalemci E., Tomsick J. A., 2006, ApJ, 642, 438

Greene J., Bailyn C. D., Orosz J. A., 2001, ApJ, 554, 1290

Greiner J. et al., 2008, PASP, 120, 405

Guillot S., Rutledge R. E., Bildsten L., Brown E. F., Pavlov G. G., Zavlin V. E., 2009, MNRAS, 392, 665

Guver T. et al., 2015, Astron. Telegram, 8149

Heida M., Jonker P. G., Torres M. A. P., Chiavassa A., 2017, ApJ, 846, 132 
Hjellming R. M., Rupen M. P., Mioduszewski A. J., 2000, in Strohmayer T. E., ed., Rossi2000: Astrophysics with the Rossi X-ray Timing Explorer Homan J., Wijnands R., Kong A., Miller J. M., Rossi S., Belloni T., Lewin W. H. G., 2006, MNRAS, 366, 235

Hynes R. I., Roche P., Walton N., Hjellming R. M., Rupen M. P., Mioduszewski A. J., 1998, IAU Circ., 6932

Hynes R. I., Roche P., Charles P. A., Coe M. J., 1999, MNRAS, 305, L49

Hynes R. I., Britt C. T., Jonker P. G., Wijnands R., Greiss S., 2012, Astron. Telegram, 4417

Ioannou Z., Robinson E. L., Welsh W. F., Haswell C. A., 2004, AJ, 127, 481

Jaisawal G. K., Homan J., Naik S., Jonker P., 2015, Astron. Telegram, 7361

Jonker P. G., 2008, in Bassa C., Wang Z., Cumming A., Kaspi V. M., eds, AIP Conf. Proc. Vol. 983, 40 Years of Pulsars: Millisecond Pulsars, Magnetars and More. Am. Inst. Phys., New York, p. 519

Jonker P. G., Nelemans G., 2004, MNRAS, 354, 355

Jonker P. G., Steeghs D., Nelemans G., van der Klis M., 2005, MNRAS, 356,621

Jonker P. G., Steeghs D., Chakrabarty D., Juett A. M., 2007, ApJ, 665, L147

Jonker P. G., Miller-Jones J. C. A., Homan J., Tomsick J., Fender R. P., Kaaret P., Markoff S., Gallo E., 2012, MNRAS, 423, 3308

Kennea J. A., Esposito P., Israel G. L., Rea N., Evans P. A., Romano P., Curran P., 2011a, Astron. Telegram, 3336

Kennea J. A. et al., 2011b, Astron. Telegram, 3613

Kennea J. A. et al., 2013, Astron. Telegram, 5479

Kennea J. A., Evans P. A., Beardmore A. P., Krimm H. A., Romano P., Yamaoka K., Serino M., Negoro H., 2017a, Astron. Telegram, 1021

Kennea J. A. et al., 2017b, Astron. Telegram, 1021

Khargharia J., Froning C. S., Robinson E. L., 2010, ApJ, 716, 1105

Khargharia J., Froning C. S., Robinson E. L., Gelino D. M., 2013, AJ, 145, 21

Kimura M. et al., 2016, Nature, 529, 54

King A. R., Kolb U., Burderi L., 1996, ApJ, 464, L127

Kitamoto S., Tsunemi H., Pedersen H., Ilovaisky S. A., van der Klis M., 1990, ApJ, 361, 590

Kong A. K. H., Jin R., Tseng C.-H., Lin E.-T., 2017, Astron. Telegram, 1024

Kosenkov I. A., Veledina A., 2018, MNRAS, 478, 4710

Kreidberg L., Bailyn C. D., Farr W. M., Kalogera V., 2012, ApJ, 757, 36

Krimm H. A. et al., 2007, Astron. Telegram, 1093

Krimm H. A. et al., 2008, Astron. Telegram, 1855

Krimm H. A., Kennea J. A., Schady P., Evans P. A., 2009, Astron. Telegram, 1893

Krimm H. A., Tomsick J. A., Markwardt C. B., Brocksopp C., Grisé F., Kaaret P., Romano P., 2011, ApJ, 735, 104

Krimm H. A. et al., 2013, ApJS, 209, 14

Krühler T. et al., 2008, ApJ, 685, 376

Kuulkers E. et al., 2007, A\&A, 466, 595

Lasota J.-P., 2000, A\&A, 360, 575

Lasota J.-P., 2008, New A Rev., 51, 752

Levine A. M., Remillard R. A., 2007, Astron. Telegram, 1024

Levine A. M., Swank J. H., Lin D., Remillard R. A., 2005a, Astron. Telegram, 578

Levine A. M., Lin D., Remillard R. A., 2005b, Astron. Telegram, 623

Lewin W., van der Klis M., 2010, Compact Stellar X-ray Sources. Cambridge Univ. Press, Cambridge, UK

MacDonald R. K. D. et al., 2014, ApJ, 784, 2

Maitra D., Cobb B., Bailyn C., Nelan J., Gonzalez D., 2005, Astron. Telegram, 628

Mandel I., 2016, MNRAS, 456, 578

Markwardt C. B. et al., 2009, Astron. Telegram, 2258

Marshall F. E., Ebisawa K., Remillard R., Valinia A., 1996, IAU Circ., 6504

Martini P., Persson S. E., Murphy D. C., Birk C., Shectman S. A., Gunnels S. M., Koch E., 2004, in Moorwood A. F. M., Iye M., eds, Proc. SPIE Vol. 5492, Ground-based Instrumentation for Astronomy. SPIE, Bellingham, p. 1653

Mata Sánchez D., Muñoz-Darias T., Casares J., Corral-Santana J. M., Shahbaz T., 2015, MNRAS, 454, 2199

Mata Sánchez D., Charles P. A., Armas Padilla M., Buckley D. A. H., Israel G. L., Linares M., Muñoz-Darias T., 2017, MNRAS, 468, 564
Matsuoka M. et al., 2009, PASJ, 61, 999

McLean I. S. et al., 2010, in McLean I. S., Ramsay S. K., Takami H., eds, Proc. SPIE, Vol. 7735, Ground-based and Airborne Instrumentation for Astronomy III. SPIE, Bellingham, p. 77351E

McLean I. S. et al., 2012, in McLean I. S., Ramsay S. K., Takami H., eds, Proc. SPIE Conf. Ser. Vol. 8446, Ground-based and Airborne Instrumentation for Astronomy IV. SPIE, Bellingham, p. 84460J

Miller-Jones J. C. A., Jonker P. G., Dhawan V., Brisken W., Rupen M. P., Nelemans G., Gallo E., 2009, ApJ, 706, L230

Miller-Jones J. C. A., Jonker P. G., Ratti E. M., Torres M. A. P., Brocksopp C., Yang J., Morrell N. I., 2011a, MNRAS, 415, 306

Miller-Jones J. C. A., Madej O. K., Jonker P. G., Homan J., Ratti E. M., Torres M. A. P., 2011b, Astron. Telegram, 3358

Miller J. M., 2001, American Astronomical Society Meeting Abstracts, p. 569

Miniutti G., Fabian A. C., Miller J. M., 2004, MNRAS, 351, 466

Minniti D. et al., 2010, New Astron., 15, 433

Molkov S. V., Lutovinov A. A., Postnov K. A., Cherepashchuk A. M., 2015, Astron. Telegram, 7520

Muñoz-Darias T., Motta S., Pawar D., Belloni T. M., Campana S., Bhattacharya D., 2010, MNRAS, 404, L94

Muñoz-Darias T. et al., 2013, MNRAS, 432, 1133

Muñoz-Darias T., Fender R. P., Motta S. E., Belloni T. M., 2014, MNRAS, 443,3270

Munoz-Darias T., Jimenez-Ibarra F., Mata Sanchez D., Armas Padilla M., Casares J., Charles P. A., 2017, Astron. Telegram, 1022

Nakahira S. et al., 2010, PASJ, 62, L27

Nakahira S. et al., 2013, Astron. Telegram, 5474

Negoro H. et al., 2011a, Astron. Telegram, 3330

Negoro H. et al., 2011b, Astron. Telegram, 3611

Negoro H. et al., 2015a, Astron. Telegram, 7504

Negoro H. et al., 2015b, Astron. Telegram, 7579

Negoro H. et al., 2017, Astron. Telegram, 1020

Orosz J. A., 2003, in van der Hucht K., Herrero A., Esteban C., eds, Proc. IAU Symp. 212, A Massive Star Odyssey: From Main Sequence to Supernova. Astron. Soc. Pac., San Francisco, p. 365

Orosz J. A., Jain R. K., Bailyn C. D., McClintock J. E., Remillard R. A., 1998, ApJ, 499, 375

Orosz J. A. et al., 2001, ApJ, 555, 489

Orosz J. A. et al., 2002, ApJ, 568, 845

Orosz J. A., McClintock J. E., Remillard R. A., Corbel S., 2004, ApJ, 616, 376

Orosz J. A., Steiner J. F., McClintock J. E., Torres M. A. P., Remillard R. A., Bailyn C. D., Miller J. M., 2011a, ApJ, 730, 75

Orosz J. A., McClintock J. E., Aufdenberg J. P., Remillard R. A., Reid M. J., Narayan R., Gou L., 2011b, ApJ, 742, 84

Özel F., Psaltis D., Narayan R., McClintock J. E., 2010, ApJ, 725, 1918

Pfahl E., Rappaport S., Podsiadlowski P., 2003, ApJ, 597, 1036

Pirard J.-F. et al., 2004, in Moorwood A. F. M., Iye M., eds, Proc. SPIE Conf. Ser. 5492, Ground-based Instrumentation for Astronomy. SPIE, Bellingham, p. 1763

Plotkin R. M., Gallo E., Jonker P. G., 2013, ApJ, 773, 59

Podsiadlowski P., Rappaport S., Pfahl E. D., 2002, ApJ, 565, 1107

Ratti E. M. et al., 2012, MNRAS, 423, 2656

Rau A., Greiner J., Filgas R., 2011a, Astron. Telegram, 3140

Rau A., Greiner J., Elliott J., Olivares E F., 2011b, Astron. Telegram, 3365

Rau A., Greiner J., Sudilovsky V., 2011c, Astron. Telegram, 3619

Rau A., Greiner J., Schady P., 2012a, Astron. Telegram, 4144

Rau A., Knust F., Kann D. A., Greiner J., 2012b, Astron. Telegram, 4380

Rau A., Tanga M., Greiner J., 2013, Astron. Telegram, 5482

Rau A., Yates R., Greiner J., 2015, Astron. Telegram, 7524

Ravi V., 2017, ApJ, 851, 114

Rea N., Jonker P. G., Nelemans G., Pons J. A., Kasliwal M. M., Kulkarni S. R., Wijnands R., 2011, ApJ, 729, L21

Reid M. J., McClintock J. E., Narayan R., Gou L., Remillard R. A., Orosz J. A., 2011, ApJ, 742, 83

Reid M. J., McClintock J. E., Steiner J. F., Steeghs D., Remillard R. A., Dhawan V., Narayan R., 2014, ApJ, 796, 2 
Remillard R. et al., 1998, IAU Circ., 6920

Remillard R. A., Orosz J. A., McClintock J. E., Bailyn C. D., 1996, ApJ, 459, 226

Repetto S., Nelemans G., 2015, MNRAS, 453, 3341

Revnivtsev M. G., Zolotukhin I. Y., Meshcheryakov A. V., 2012, MNRAS, 421, 2846

Rupen M. P., Dhawan V., Mioduszewski A. J., 2005, Astron. Telegram, 589

Russell T. D., Soria R., Motch C., Pakull M. W., Torres M. A. P., Curran P. A., Jonker P. G., Miller-Jones J. C. A., 2014, MNRAS, 439, 1381

Russell T. D. et al., 2015, MNRAS, 450, 1745

Russell D. M., Fender R. P., 2008, MNRAS, 387, 713

Russell D. M., Fender R. P., Hynes R. I., Brocksopp C., Homan J., Jonker P. G., Buxton M. M., 2006, MNRAS, 371, 1334

Russell D. M., Fender R. P., Jonker P. G., 2007, MNRAS, 379, 1108

Russell D. M., Muñoz-Darias T., Lewis F., Soleri P., 2010, Astron. Telegram, 2775

Russell D. M., Lewis F., Roche P., Altamirano D., 2011, Astron. Telegram, 3359

Russell D. M. et al., 2012, Astron. Telegram, 4456

Sanna A., Altamirano D., Rushton A., Jonker P., 2015, Astron. Telegram, 7278

Schirmer M., 2013, ApJS, 209, 21

Shahbaz T., Kuulkers E., 1998, MNRAS, 295, L1

Shahbaz T., van der Hooft F., Charles P. A., Casares J., van Paradijs J., 1996, MNRAS, 282, L47

Shahbaz T., Russell D. M., Zurita C., Casares J., Corral-Santana J. M., Dhillon V. S., Marsh T. R., 2013, MNRAS, 434, 2696

Shaposhnikov N., Markwardt C., Swank J., Krimm H., 2010, ApJ, 723, 1817

Shidatsu M. et al., 2017a, ApJ, No. 850, 155

Shidatsu M. et al., 2017b, Astron. Telegram, No. 1022

Skrutskie M. F. et al., 2006, AJ, 131, 1163

Steeghs D., Torres M. A. P., Pych W., Thompson I., 2005a, Astron. Telegram, No. 585

Steeghs D., Torres M. A. P., Koviak K., McCarthy P., Jonker P. G., 2005b, Astron. Telegram, No. 629

Steeghs D., McClintock J. E., Parsons S. G., Reid M. J., Littlefair S., Dhillon V. S., 2013, ApJ, 768, 185

Sugimoto J. et al., 2015, Astron. Telegram, No. 8143

Suzuki K. et al., 2015, Astron. Telegram, No. 7233

Tachibana Y. et al., 2017, Astron. Telegram, No. 1022

Taranova O. G., Shenavrin V. I., 2001, Astron. Lett., 27, 25

Tetarenko B. E., Sivakoff G. R., Heinke C. O., Gladstone J. C., 2016a, ApJS, 222,15

Tetarenko B. E. et al., 2016b, ApJ, 825, 10
Tokunaga A. T., 2000, Infrared Astronomy. Springer-Verlag, New York, p. 143

Tomsick J. A., Kalemci E., Kaaret P., 2004, ApJ, 601, 439

Tomsick J. A., DelSanto M., Belloni T., 2012, Astron. Telegram, 4393

Torres M. A. P. et al., 2002, ApJ, 569, 423

Torres M. A. P., Callanan P. J., Garcia M. R., Zhao P., Laycock S., Kong A. K. H., 2004, ApJ, 612, 1026

Torres M. A. P., Steeghs D., Jonker P. G., Morrell N., Roth M., Kerber F., 2007, Astron. Telegram, 1072

Torres M. A. P., Jonker P. G., Steeghs D., Chen H.-W., Shectman S. A., Floyd D. J. E., 2009a, Astron. Telegram, 1958

Torres M. A. P., Jonker P. G., Steeghs D., Yan H., Huang J., Soderberg A. M., 2009b, Astron. Telegram, 2263

Torres M. A. P., Steeghs D., Jonker P. G., Thompson I., Soderberg A. M., 2009c, Astron. Telegram, 2268

Torres M. A. P., Jonker P. G., Miller-Jones J. C. A., Steeghs D., Repetto S., Wu J., 2015, MNRAS, 450, 4292

Turler M., Cadolle Bel M., Diehl R., Westergaard N.-J., McBreen B., Williams O. R., Grebenev S. A., Lutovinov A., 2005, Astron. Telegram, 624

Usui R. et al., 2012, Astron. Telegram, 4140

van der Hooft F., Heemskerk M. H. M., Alberts F., van Paradijs J., 1998, A\&A, 329, 538

van Grunsven T. F. J., Jonker P. G., Verbunt F. W. M., Robinson E. L., 2017, MNRAS, 472, 1907

van Paradijs J., McClintock J. E., 1994, A\&A, 290, 133

van Paradijs J., White N., 1995, ApJ, 447, L33

Webb N. A., Naylor T., Ioannou Z., Charles P. A., Shahbaz T., 2000, MNRAS, 317, 528

Wu Y. X., Yu W., Li T. P., Maccarone T. J., Li X. D., 2010, ApJ, 718, 620

Yuan H. B., Liu X. W., Xiang M. S., 2013, MNRAS, 430, 2188

Zacharias N., Finch C. T., Girard T. M., Henden A., Bartlett J. L., Monet D. G., Zacharias M. I., 2013, AJ, 145, 44

Zdziarski A. A., Ziółkowski J., Bozzo E., Pjanka P., 2016, A\&A, 595, A52

Zurita Heras J. A., Chaty S., Cadolle Bel M., Prat L., 2011, MNRAS, 413, 235

Zurita C. et al., 2002, MNRAS, 334, 999

Zurita C., Casares J., Hynes R. I., Shahbaz T., Charles P. A., Pavlenko E. P., 2004, MNRAS, 352, 877

APPENDIX: QUIESCENT DETECTIONS 
Table A1. BHXBs with values of outburst and quiescence magnitudes, orbital periods and inclination angles, represented graphically in Figs 4(a) and (b). The well-known BHXB GRS 1915+105 is not included in this table, as it has never been observed in quiescence.

\begin{tabular}{|c|c|c|c|c|c|}
\hline BHXB & Filter & $\begin{array}{l}\text { AB Magnitt } \\
\text { Outburst } \\
\text { (mag) }\end{array}$ & $\begin{array}{l}\text { Quiescence } \\
\quad(\mathrm{mag})\end{array}$ & $\begin{array}{l}\text { Orbital } \\
\text { period } \\
\text { (h) }\end{array}$ & $\begin{array}{l}\text { Inclination } \\
\text { angle } \\
\left({ }^{\circ}\right)\end{array}$ \\
\hline XTE J1118+480 & $K_{s}$ & $12.64 \pm 0.09^{b}$ & $18.45^{l}$ & $4.078414 \pm 0.000005^{r}$ & $68 \leq i \leq 79^{z}$ \\
\hline SAX J1819.3-2525 & $K_{s}$ & $14.4 \pm 0.3^{c}$ & $14.68 \pm 0.05^{m}$ & $67.6152 \pm 0.0002^{s}$ & $72.3 \pm 4.1^{a a}$ \\
\hline XTE J1550-564 & $K_{s}$ & $13.91 \pm 0.09^{d}$ & $18.00 \pm 0.04^{n}$ & $37.0088 \pm 0.0001^{n}$ & $75 \pm 4^{n}$ \\
\hline GRO J1655-40 & $K_{s}$ & $14.25^{e}$ & $15.12^{\circ}$ & $62.920 \pm 0.003^{t}$ & $69 \pm 2^{a b}$ \\
\hline GX 339-4 & $K_{s}$ & $12.41 \pm 0.01^{g}$ & $15.2 \pm 0.3^{g}$ & $42.2088 \pm 0.0001^{v}$ & $37 \leq i \leq 78^{v}$ \\
\hline IGR J17451-3022 & $K_{s}$ & $17.39 \pm 0.16^{h}$ & $17.59 \pm 0.14^{h}$ & $6.284 \pm 0.001^{w}$ & $71 \leq i \leq 76^{a d}$ \\
\hline MAXI J1836-194‡ & $K_{s}$ & $14.00 \pm 0.07^{i}$ & $20.9 \pm 0.3^{h}$ & $<4.9^{x}$ & $4 \leq i \leq 15^{x}$ \\
\hline XTE J1650-500‡ & $K_{s}$ & $15.14 \pm 0.13^{j}$ & $>17.8^{h}$ & $7.60 \pm 0.02^{y}$ & $>47^{y}$ \\
\hline GRO J0422+32* & $V$ & $13.2 \pm 0.1$ & $22.4 \pm 0.3$ & $5.09 \pm 0.01$ & $10 \leq i \leq 50^{a e}$ \\
\hline A $0620-00^{*}$ & $V$ & 11.22 & 18.32 & $7.7235 \pm 0.0001$ & $54.1 \pm 1.1^{a f}$ \\
\hline GRO J1655-40* & $V$ & 14.02 & 17.22 & $62.920 \pm 0.003^{t}$ & $69 \pm 2^{a k}$ \\
\hline GS $2023+338^{*}$ & $V$ & $11^{a l}$ & $18.44 \pm 0.02$ & $155.311 \pm 0.002^{u}$ & $67_{-1}^{+3 a c}$ \\
\hline GX 339-4 & $V$ & $14.76 \pm 0.02^{\mathrm{am}}$ & $19.67 \pm 0.06^{a n}$ & $42.2088 \pm 0.0001^{v}$ & $37 \leq i \leq 78^{v}$ \\
\hline $4 U 1543-475$ & $V$ & $14.9^{a o}$ & $16.6 \pm 0.1^{a p}$ & $26.79377 \pm 0.00007^{a q}$ & $20.7 \pm 1.5^{a q}$ \\
\hline SAX J1819.3-2525 & $V$ & $8.8^{a r}$ & $13.96 \pm 0.02^{a s}$ & $67.6152 \pm 0.0002^{s}$ & $72.3 \pm 4.1^{a a}$ \\
\hline XTE J1118+480 & $V$ & $13.0 \pm 0.3^{a t}$ & $19.6^{l}$ & $4.078414 \pm 0.000006^{r}$ & $68 \leq i \leq 79^{z}$ \\
\hline XTE J1550-564 & $V$ & $16.6^{a u}$ & $22.0 \pm 0.4^{a u}$ & $37.0088 \pm 0.0001^{n}$ & $75 \pm 4^{n}$ \\
\hline GS $1354-64$ & $V$ & $16.9^{a v}$ & $21.5^{a w}$ & $61.068 \pm 0.002^{a w}$ & $\leq 79^{a w}$ \\
\hline XTE J1859+226 & $V$ & $15.3 \pm 0.1^{a x}$ & $23.3 \pm 0.1^{a x}$ & $6.58^{a y}$ & $\leq 70^{a y}$ \\
\hline
\end{tabular}

Ref: ${ }^{a}$ Rau, Greiner \& Filgas (2011a), ${ }^{b}$ Taranova \& Shenavrin (2001), ${ }^{c}$ Chaty et al. (2003), ${ }^{d}$ Curran \& Chaty (2013), ${ }^{e}$ Buxton, Bailyn \& Maitra (2005), ${ }^{f}$ Garner et al. (2015), ${ }^{g}$ Chaty et al. (2002), ${ }^{h}$ this work, ${ }^{i}$ Rau et al. (2011c), ${ }^{j}$ Curran et al. (2012), ${ }^{k}$ Shahbaz et al. (2013), ${ }^{l}$ Gelino et al. (2006), ${ }^{m}$ MacDonald et al. (2014), ${ }^{n}$ Orosz et al. (2011a), ${ }^{o}$ Greene, Bailyn \& Orosz (2001), ${ }^{p}$ Zurita et al. (2004), ${ }^{q}$ Corral-Santana et al. (2013b), ${ }^{r}$ Torres et al. (2004), ${ }^{s}$ Corral-Santana et al. (2011), ${ }^{t}$ van der Hooft et al. (1998), ${ }^{u}$ Casares, Charles \& Naylor (1992), Heida et al. (2017), ${ }^{w}$ Jaisawal et al. (2015), ${ }^{x}$ Russell et al. (2014), ${ }^{y}$ Orosz et al. (2004), ${ }^{z}$ Khargharia et al. (2013), ${ }^{a}$ MacDonald et al. (2014), ${ }^{a b}$ Beer \& Podsiadlowski (2002), ${ }^{a c}$ Khargharia, Froning \& Robinson (2010), ${ }^{a d}$ Zdziarski et al. (2016), ${ }^{a e}$ Webb et al. (2000), ${ }^{a f}$ van Grunsven et al. (2017), ${ }^{a g}$ Shahbaz et al. (1996), ${ }^{a h}$ Gelino, Harrison \& McNamara (2001), ${ }^{a i}$ Remillard et al. (1996), ${ }^{a j}$ Ioannou et al. (2004), ${ }^{a k}$ Beer \& Podsiadlowski (2002), ${ }^{a l}$ Kimura et al. (2016), ${ }^{a m}$ Buxton et al. (2012), ${ }^{a n}$ Kosenkov \& Veledina (2018), ${ }^{a o}$ Blissett et al. (1983), ${ }^{a p}$ Orosz et al. (1998), ${ }^{a q}$ Orosz (2003), ${ }^{a r}$ Orosz et al. (2001), ${ }^{a s}$ MacDonald et al. (2014), ${ }^{a t}$ Torres et al. (2002), ${ }^{a u}$ Orosz et al. (2002), ${ }^{a v}$ Kitamoto et al. (1990), ${ }^{a w}$ Casares et al. (2009), ${ }^{a x}$ Zurita et al. (2002) and ${ }^{a y}$ Corral-Santana et al. (2013a). ‡Sources in our sample. * Magnitudes and orbital periods are taken from Shahbaz \& Kuulkers (1998) and references therein, unless indicated otherwise. 


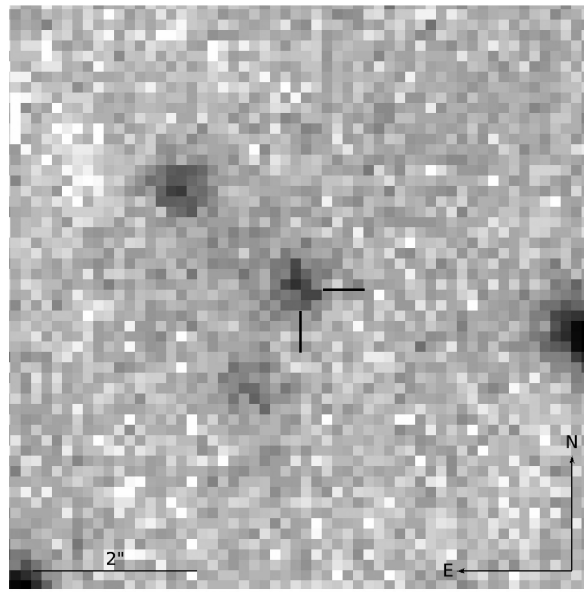

(a) XTE J1726-476

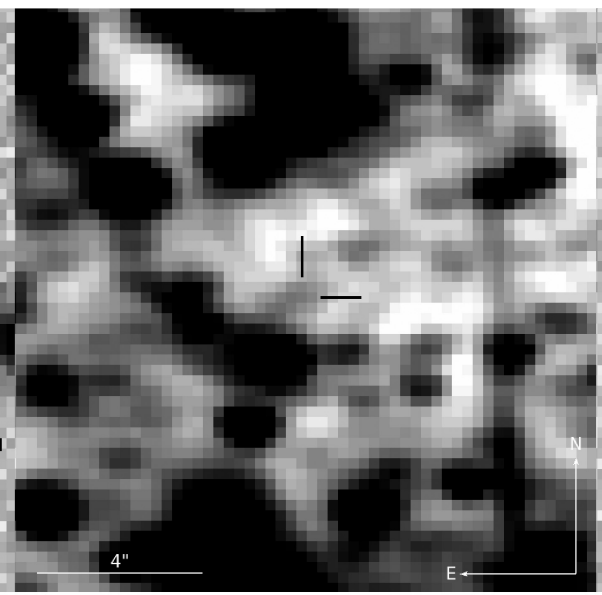

(b) Swift J174510.8-262411

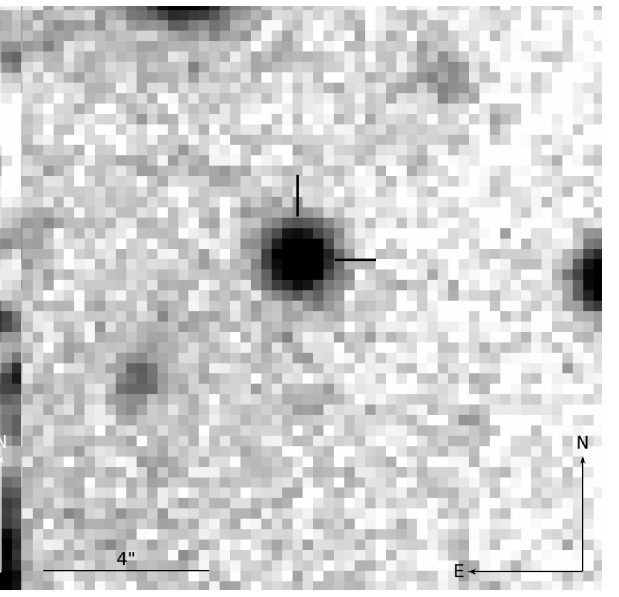

(c) MAXI J1807+132, $i^{\prime}$-band

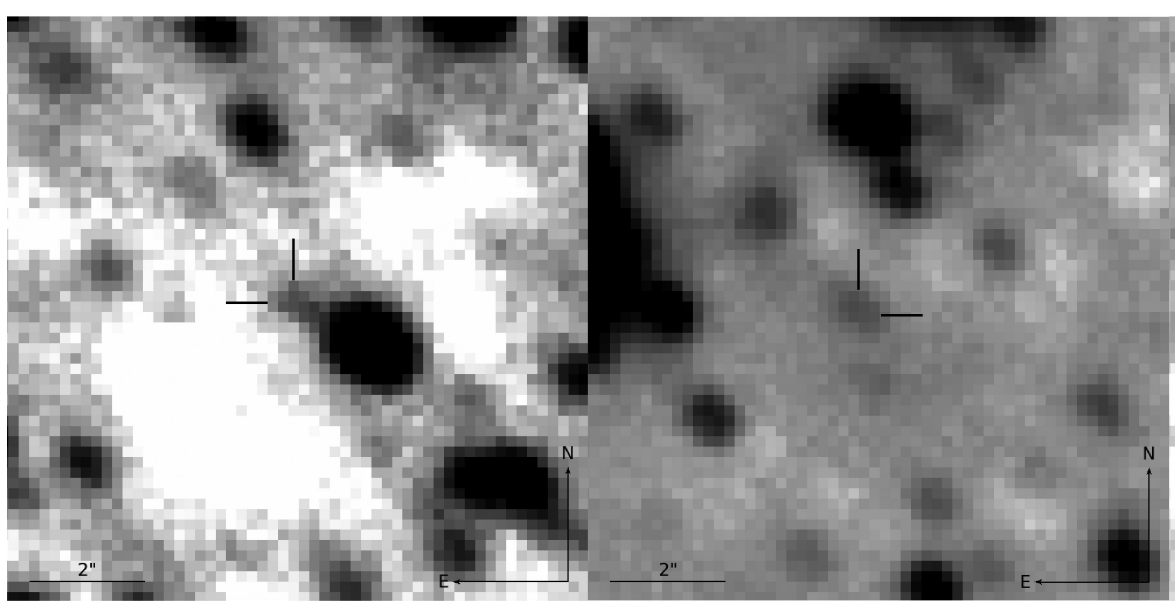

(d) MAXI J1828-249

(e) MAXI J1836-194

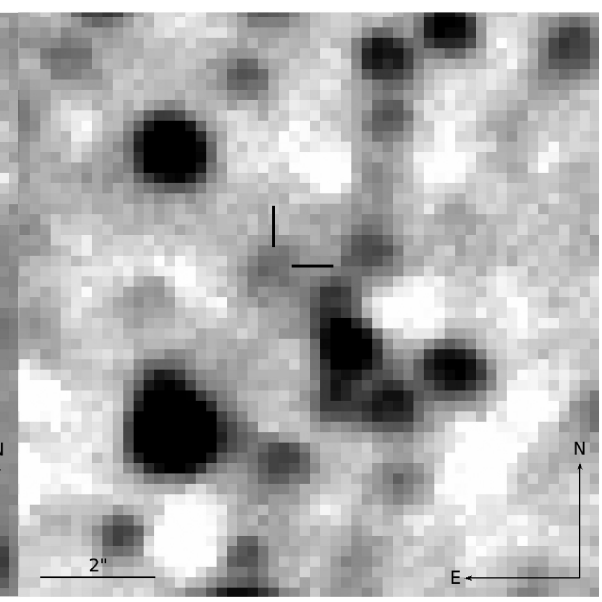

(f) XTE J1856+053

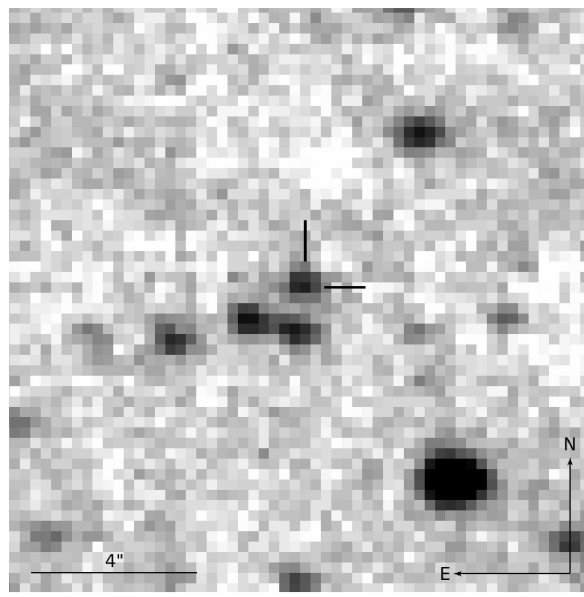

(g) Swift J1910.2-0546

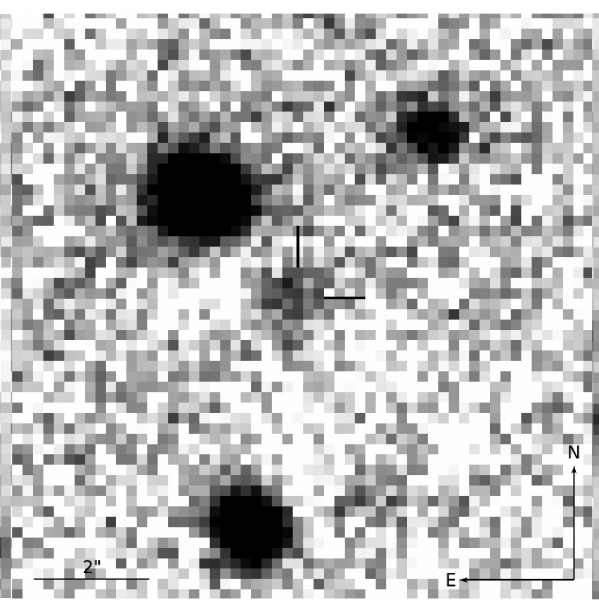

(h) MAXI J1957+032

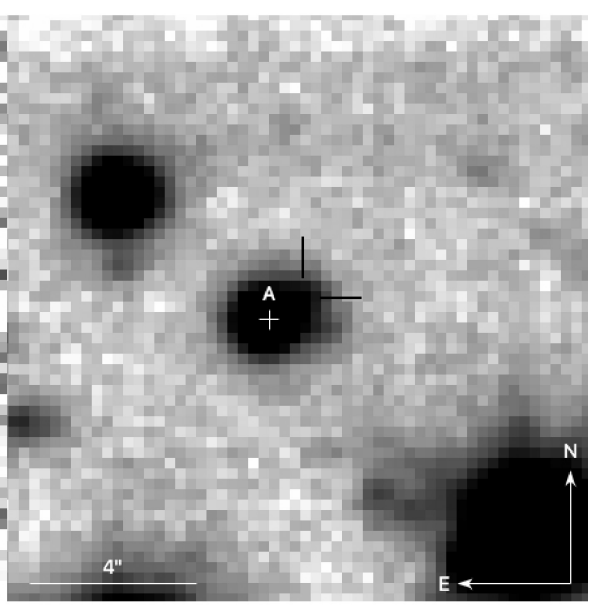

(i) XTE J2012+381

Figure A1. Finder charts of the XRBs with a quiescent counterpart detected. These sources have a known position for the counterpart in outburst. All images are in the NIR $K_{\mathrm{S}}$ band, unless indicated otherwise. The position of the detected counterparts is indicated in every image. For image (i), we also indicate the other star identified by Hynes et al. (1999) and label it A. 


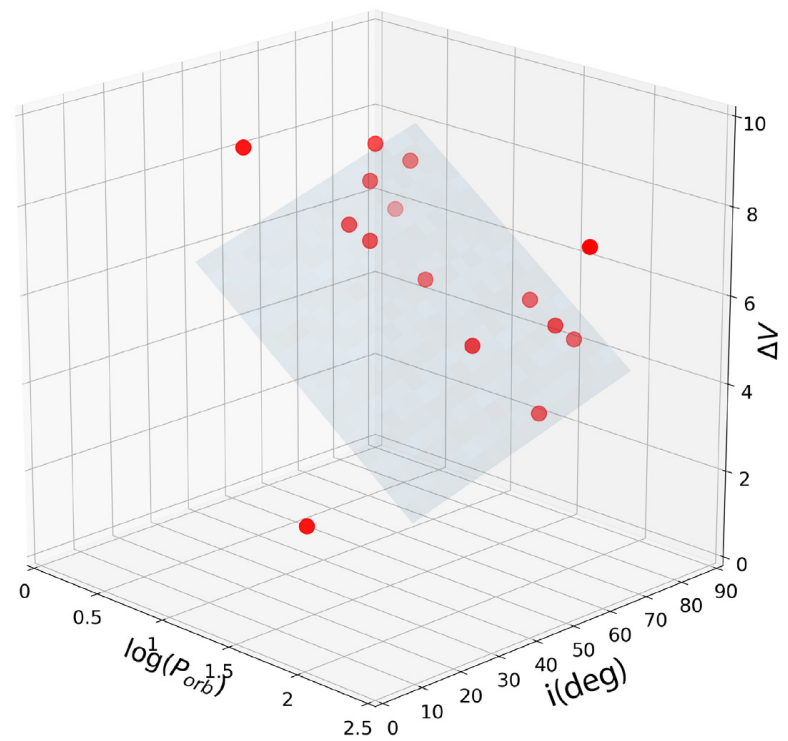

(a)

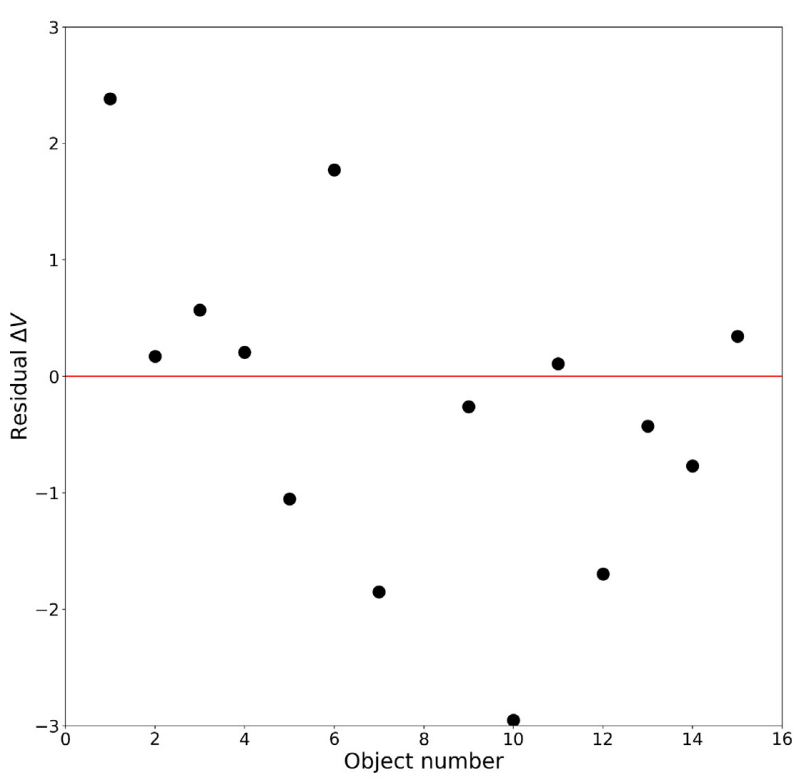

(c)

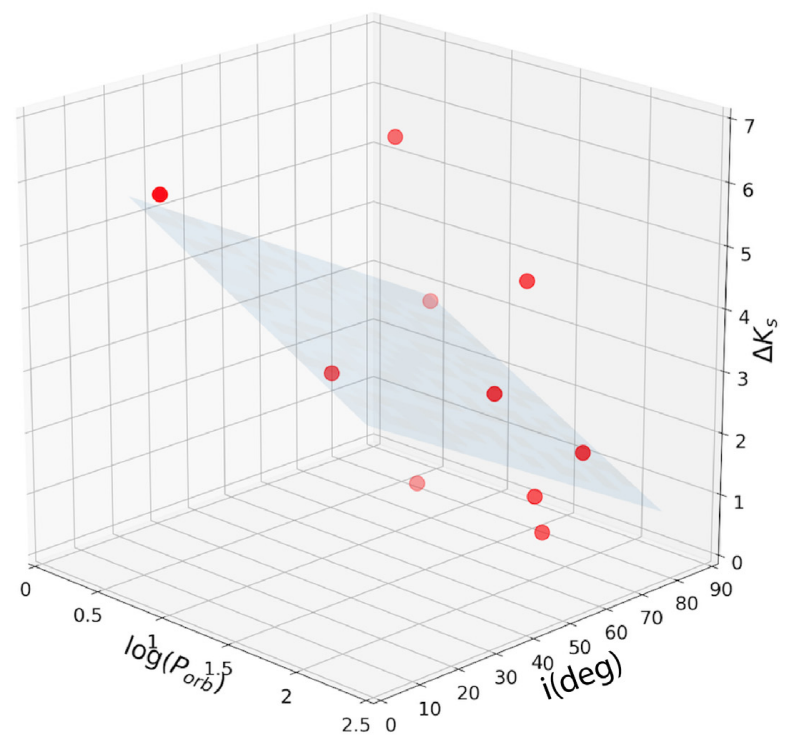

(b)

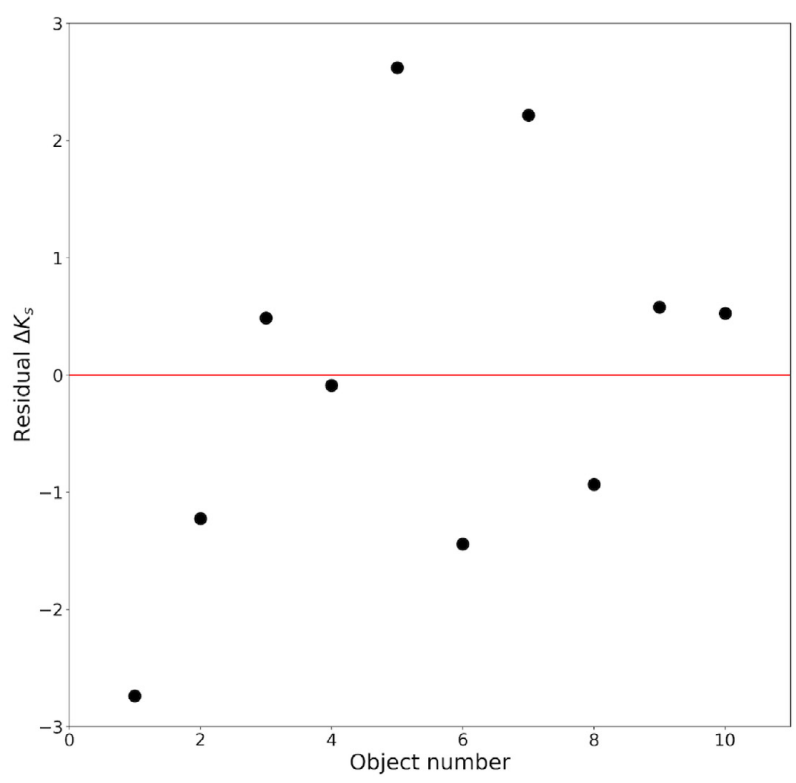

(d)

Figure A2. (a) 3D least squares plane fit to the $V$-band outburst amplitude versus the logarithm of the orbital period $P_{\text {orb }}$ and the inclination angle $i$ (for references see Table A1); adapted from Shahbaz \& Kuulkers (1998). (b) Same as for panel (a) but now for the $K_{s}$-band amplitude. Residuals of the fit for (c) $\Delta V$ and (d) $\Delta K_{s}$.

This paper has been typeset from a $\mathrm{T}_{\mathrm{E}} \mathrm{X} / \mathrm{LT} \mathrm{E} \mathrm{X}$ file prepared by the author. 